PREPARED FOR SUBMISSION TO JCAP

\title{
Generalized spin-dependent WIMP-nucleus interactions and the DAMA modulation effect
}

\author{
Stefano Scopel, ${ }^{a}$ Kook-Hyun Yoon ${ }^{b}$ Jong-Hyun Yoon ${ }^{c}$ \\ Department of Physics, Sogang University, Seoul, South Korea \\ E-mail: scopel@sogang.ac.kr, koreasds@naver.com, pledge200@gmail.com
}

\begin{abstract}
Guided by non-relativistic Effective Field Theory (EFT) we classify the most general spin-dependent interactions between a fermionic Weakly Interacting Massive Particle (WIMP) and nuclei, and within this class of models we discuss the viability of an interpretation of the DAMA modulation result in terms of a signal from WIMP elastic scatterings using a halo-independent approach. We find that, although several relativistic EFT's can lead to a spin-dependent cross section, in some cases with an explicit, non-negligible dependence on the WIMP incoming velocity, three main scenarios can be singled out in the non-relativistic limit which approximately encompass them all, and that only differ by their dependence on the transferred momentum. For two of them compatibility between DAMA and other constraints is possible for a WIMP mass below $30 \mathrm{GeV}$, but only for a WIMP velocity distribution in the halo of our Galaxy which departs from a Maxwellian. This is achieved by combining a suppression of the WIMP effective coupling to neutrons (to evade constraints from xenon and germanium detectors) to an explicit quadratic or quartic dependence of the cross section on the transferred momentum (that leads to a relative enhancement of the expected rate off sodium in DAMA compared to that off fluorine in droplet detectors and bubble chambers). For larger WIMP masses the same scenarios are excluded by scatterings off iodine in COUPP.
\end{abstract}




\section{Contents}

1 Introduction $\quad 1$

2 Generalized spin-dependent interactions $\quad 4$

$\begin{array}{lll}3 & \text { WIMP direct detection rate } & 7\end{array}$

4 Halo-independent analysis $\quad 8$

5 Constraints from direct detection experiments and compatibility factor 10

6 Results $\quad 12$

$\begin{array}{lll}7 & \text { Conclusions } & 15\end{array}$

$\begin{array}{ll}\text { A WIMP response functions } & 18\end{array}$

$\begin{array}{lll}\text { B Experimental inputs for the analysis } & 18\end{array}$

$\begin{array}{ll}\text { C Nuclear response functions for chlorine } & 21\end{array}$

\section{Introduction}

Many underground experiments are currently searching for Weakly Interacting Massive Particles (WIMPs), which are the most popular candidates to provide the Dark Matter (DM) which is believed to make up $27 \%$ of the total mass density of the Universe[1], and more than $90 \%$ of the halo of our Galaxy. One of them (DAMA[2]) has been observing for more than 15 years a yearly modulation effect in the low part of its energy spectrum which is consistent with that expected due to the Earth rotation around the Sun from the elastic scattering of WIMPs off the sodium iodide nuclei that constitute the crystals of its scintillators. Many experimental collaborations using nuclear targets different from NaI and various background-subtraction techniques to look for WIMP-elastic scattering (LUX[3], XENOn100[4], XENON10[5], KIMS[6, 7], CDMS-Ge[8], CDMSlite [9], SuperCDMS[10], SIMPLE[11], COUPP[12], PICASSO[13], PICO-2L[14]) have failed to observe any anomaly so far, implying severe constraints on the most popular WIMP scenarios used to explain the DAMA excess. This is particularly compelling when the KIMS bound [6] (which is obtained using $C s I$ crystals) is taken at face value, and when the WIMP mass is large enough to rule out the possibility that the DAMA effect is mainly due to WIMP scatterings on sodium. In that case both DAMA and KIMS should observe the same WIMP-iodine process and no room is left to model building to reconcile the discrepancy (although several experimental uncertainties might still be advocated to question the robustness of the KIMS bound ${ }^{1}$ ). However, when the WIMP mass is small enough it is possible to assume that the contribution of

\footnotetext{
${ }^{1}$ The raw background of KIMS is about a factor of three larger than that of DAMA, so that KIMS sensitivity relies on background subtraction techniques that can potentially introduce systematic errors. Moreover KIMS has recently published a new measurement of its quenching factor [15] significantly smaller than that used for its published bounds $[6,7]$ and reducing its sensitivity to WIMPs scatterings at low energy.
} 
WIMP-iodine scatterings in the expected event rates of both DAMA and KIMS vanishes and that the DAMA effect is due to scatterings on sodium. In this case the constraints on DAMA can be potentially relaxed by considering models where the expected WIMP scattering rate on sodium is enhanced compared to that off the nuclei used to obtain the experimental bounds.

Moreover, the energy dependence of the WIMP-induced scattering spectrum depends on the velocity distribution $f(\vec{v})$ of the incoming WIMPs. Historically, for the latter the isothermal sphere model has been assumed, i.e. a thermalized non-relativistic gas described by a Maxwellian distribution whose temperature $v_{r m s} \simeq 270 \mathrm{~km} / \mathrm{sec}$ is determined from the galactic rotational velocity by assuming equilibrium between gravitational attraction and WIMP pressure. However, while the presence of a thermalized component in the WIMP halo of or Galaxy is confirmed by numerical simulations the detailed merger history of the Milky Way is not known, allowing for the possibility of the presence of sizeable non-thermal components for which the density, direction and speed of WIMPs are hard to predict.

In light of the situation summarized above several new directions have been explored in the recent past both to remove as much as possible the dependence on specific theoretical assumptions (both of particle-physics and astrophysical origin) from the analysis of DM direct detection data and to extend its scope to a wider class of models. Starting from [16] a general strategy has been developed [17-19] to factor out the dependence on $f(\vec{v})$ of the expected WIMP-nucleus differential rate $d R / d E_{R}$ at the given recoil energy $E_{R}$. This approach exploits the fact that $d R / d E_{R}$ depends on $f(\vec{v})$ only through the minimal speed $v_{\text {min }}$ that the WIMP must have to deposit at least $E_{R}$, i.e.:

$$
\frac{d R}{d E_{R}} \propto \eta\left(v_{\min }\right) \equiv \int_{|\vec{v}|>v_{\min }} \frac{f(\vec{v})}{|\vec{v}|} d^{3} v
$$

By mapping recoil energies $E_{R}$ into same ranges of $v_{\text {min }}$ the dependence on $\eta\left(v_{\text {min }}\right)$ and so on $f(\vec{v})$ cancels out in the ratio of expected rates on different targets. Since the mapping between $E_{R}$ and $v_{\min }$ depends on the nuclear mass the factorization of $\eta\left(v_{\min }\right)$ is only possible in the case of detectors with a single nuclear target, or for which the expected rate is dominated by scatterings on a single target. However in the following we will extend this procedure to check the compatibility between a candidate signal (such as that from the DAMA experiment) and a null experiment also in the case when the latter contains different targets.

On the particle-physics side one of the most popular scenarios for WIMP-nucleus scattering is a spin-dependent interaction where the WIMP particle $\chi$ is a fermion (either Dirac or Majorana) that recoils on the target nucleus $T$ through it coupling to the spin $\vec{S}_{N}$ of nucleons $N=(p, n)$ :

$$
\mathcal{L}_{i n t} \propto \vec{S}_{\chi} \cdot \vec{S}_{N}=c^{p} \vec{S}_{\chi} \cdot \vec{S}_{p}+c^{n} \vec{S}_{\chi} \cdot \vec{S}_{n}
$$

The above effective Lagrangian can arise as the low-energy limit of a relativistic axial coupling $\bar{\chi} \gamma_{5} \gamma^{\mu} \chi \bar{N} \gamma_{5} \gamma^{\mu} N$ (the incoming WIMP velocity in the halo of our Galaxy is expected to be of order $10^{-3} \mathrm{c}$ ). One of the main motivations of such scenario is the fact that the most stringent bounds on the interpretation of the DAMA effect in terms of WIMPnuclei scatterings arise today from detectors using xenon (LUX[3], XENON100[4]) and germanium (SuperCDMS[10]) whose spin is mostly originated by an unpaired neutron, while both sodium and iodine in DAMA have an unpaired proton: as already pointed out by some authors[20, 21], if the WIMP effective coupling to neutrons $c^{n}$ is suppressed compared to that on protons $c^{p}$ this class of bounds can be evaded. However this scenario is 
presently constrained by droplet detectors (SIMPLE[11], COUPP[12]) and bubble chambers (PICASSO[13], PICO-2L[14]) which all use nuclear targets with an unpaired proton (in particular, they all contain ${ }^{19} \mathrm{~F}$, while SIMPLE contains also ${ }^{35} \mathrm{Cl}$ and ${ }^{37} \mathrm{Cl}$ and COUPP uses also ${ }^{127} I$ ). As a consequence, this class of experiments have been shown to rule out the scenario of Eq. (1.2) also for $c^{n} \ll c^{p}$ when standard assumptions are made on the WIMP local density and velocity distribution in our Galaxy[14, 21].

The spin-dependent scenario has been further extended in Refs. [22, 23], where a generalization of the coupling of Eq.(1.2) has been discussed. In this scenario the WIMP particle couples to nucleons through a relativistic axial coupling $\bar{\chi} \gamma_{5} \chi \bar{N} \gamma_{5} N$ through the exchange of a light pseudoscalar, leading to a non-relativistic coupling $\left(\vec{S}_{\chi} \cdot \vec{q}\right)\left(\vec{S}_{N} \cdot \vec{q}\right)$ which depends on the projection of the nuclear spin component along the transferred momentum $\vec{q}$. This scenario, which combines a spin-dependent nuclear response function mainly sensitive to unpaired nucleons (leading to relaxed constraints from $G e$ and $X e$ detectors on DAMA when $c^{n} \ll c^{p}$ ) to a non-standard dependence on the transferred momentum $\vec{q}$, has also been shown to be in tension with bubble chambers and droplet detectors, albeit to a lesser extent, at least for standard assumptions on the WIMP velocity distribution [21].

In the present paper we wish to elaborate further on the possibility that the nonrelativistic effective coupling between WIMPS and nuclei is due to a combination of a spindependent nuclear response function and a generalized momentum dependence. In particular, we wish to assess the viability of this kind of scenario by departing from the Isothermal Sphere model and adopting the previously mentioned halo-independent approach.

Our main motivation is the simple observation that models such as that of Refs. [22, 23], where the WIMP-nucleus cross section depends on additional factors $q^{n}$ (with $n>0$ ) of the transferred momentum, tend to suppress the sensitivity to the DAMA effect of droplet detectors and bubble chambers because, due to a combination of low energy thresholds and light target masses, a critical fraction of the WIMP-scattering events expected in the latter have transferred momenta below those related to the DAMA effect. For this reason, scenarios that combine a spin-dependent coupling mostly to protons to additional factors $q^{n}$ in the cross section are expected to have better chances to reconcile the DAMA effect to the constraints from all other detectors. This will be confirmed in our analysis.

In order to develop this program in a systematic way, we will rely on the analysis of Ref.[24], where the most general non-relativistic Hamiltonian $\mathrm{H}$ describing the elastic scattering of a fermionic WIMP particle off nuclei was written in terms of the sum of all the terms invariant by Galilean transformations. Using this approach, we will single out all the possible spin-dependent interactions compatible to Galilean invariance that will be the subject of our phenomenological analysis.

Our paper is organized as follows. In Section 2 we use the non-relativistic Effective Field Theory approach of Ref.[24] to single out the generalized spin-dependent interactions we wish to discuss in the rest of our paper, and set our notations; in Section 3 we provide the formulas to calculate expected rates for WIMP-nucleus scattering in our scenarios; in Section 4 we summarize the main ingredients for the halo-independent analysis; in Section 5 we introduce a compatibility factor between DAMA and other experiments and provide a recipe to extend it to the case of constraints involving more than one nuclear target. The results of our quantitative analysis are given in Section 6 , while Section 7 is devoted to our conclusions. Finally, in the Appendices we provide some technical details of our procedure and summarize the experimental inputs used in the analysis. 


\section{Generalized spin-dependent interactions}

The most general WIMP-nucleus spin-dependent interactions can be single out by making use of the non-relativistic EFT approach of Ref.[24]. According to that analysis the most general Hamiltonian density for the process can be expressed in terms of a combination of the following five Hermitian operators, which act on the two-particle Hilbert space spanned by tensor products of WIMP and nucleon states:

$$
1_{\chi N} \quad i \vec{q} \quad \vec{v}^{\perp} \quad \vec{S}_{\chi} \quad \vec{S}_{N},
$$

where $1_{\chi N}$ is the identity operator, $\vec{q}$ is the transferred momentum, $\vec{S}_{\chi}$ and $\vec{S}_{N}$ are the WIMP and nucleon spins, respectively, while $\vec{v}^{\perp}=\vec{v}+\frac{\vec{q}}{2 \mu_{\chi N}}$ (with $\mu_{\chi N}$ the WIMP-nucleon reduced mass) is the relative transverse velocity operator satisfying $\vec{v}^{\perp} \cdot \vec{q}=0$. Including terms that are at most linear in $\vec{S}_{N}, \vec{S}_{\chi}$ and $\vec{v}^{\perp}$ only the following 15 non-relativistic quantum mechanical operators can be constructed out of (2.1):

$$
\begin{aligned}
\mathcal{O}_{1} & =1_{\chi} 1_{N} ; \quad \mathcal{O}_{2}=\left(v^{\perp}\right)^{2} ; \quad \mathcal{O}_{3}=i \vec{S}_{N} \cdot\left(\frac{\vec{q}}{m_{N}} \times \vec{v}^{\perp}\right) \\
\mathcal{O}_{4} & =\vec{S}_{\chi} \cdot \vec{S}_{N} ; \quad \mathcal{O}_{5}=i \vec{S}_{\chi} \cdot\left(\frac{\vec{q}}{m_{N}} \times \vec{v}^{\perp}\right) ; \quad \mathcal{O}_{6}=\left(\vec{S}_{\chi} \cdot \frac{\vec{q}}{m_{N}}\right)\left(\vec{S}_{N} \cdot \frac{\vec{q}}{m_{N}}\right) \\
\mathcal{O}_{7} & =\vec{S}_{N} \cdot \vec{v}^{\perp} ; \quad \mathcal{O}_{8}=\vec{S}_{\chi} \cdot \vec{v}^{\perp} ; \quad \mathcal{O}_{9}=i \vec{S}_{\chi} \cdot\left(\vec{S}_{N} \times \frac{\vec{q}}{m_{N}}\right) \\
\mathcal{O}_{10} & =i \vec{S}_{N} \cdot \frac{\vec{q}}{m_{N}} ; \quad \mathcal{O}_{11}=i \vec{S}_{\chi} \cdot \frac{\vec{q}}{m_{N}} ; \quad \mathcal{O}_{12}=\vec{S}_{\chi} \cdot\left(\vec{S}_{N} \times \vec{v}^{\perp}\right) \\
\mathcal{O}_{13} & =i\left(\vec{S}_{\chi} \cdot \vec{v}^{\perp}\right)\left(\vec{S}_{N} \cdot \frac{\vec{q}}{m_{N}}\right) ; \quad \mathcal{O}_{14}=i\left(\vec{S}_{\chi} \cdot \frac{\vec{q}}{m_{N}}\right)\left(\vec{S}_{N} \cdot \vec{v}^{\perp}\right) \\
\mathcal{O}_{15} & =-\left(\vec{S}_{\chi} \cdot \frac{\vec{q}}{m_{N}}\right)\left(\left(\vec{S}_{N} \times \vec{v}^{\perp}\right) \cdot \frac{\vec{q}}{m_{N}}\right),
\end{aligned}
$$

so that the the most general Hamiltonian density describing the WIMP-nucleus interaction can be written as:

$$
\mathcal{H}(\mathbf{r})=\sum_{\tau=0,1} \sum_{k=1}^{15} c_{k}^{\tau} \mathcal{O}_{k}(\mathbf{r}) t^{\tau}
$$

where $t^{0}=1, t^{1}=\tau_{3}$ denote the the $2 \times 2$ identity and third Pauli matrix in isospin space, respectively, and the isoscalar and isovector (dimension -2) coupling constants $c_{k}^{0}$ and $c_{k}^{1}$, are related to those to protons and neutrons $c_{k}^{p}$ and $c_{k}^{n}$ by $c_{k}^{p}=\left(c_{k}^{0}+c_{k}^{1}\right) / 2$ and $c_{k}^{n}=\left(c_{k}^{0}-c_{k}^{1}\right) / 2$.

The basic assumption of Ref.[24] is that the nuclear interaction is the sum of the interactions of the WIMPs with the individual nucleons in the nucleus, and so neglects any effect involving two or more nucleons. Notice that while such effects are usually expected to be small, they can become non-negligible in particular circumstances, such as when the leading terms is suppressed by a cancellation between the $c_{k}^{p}$ and $c_{k}^{n}$ couplings $[25,26]$. With this simplification the WIMP scattering amplitude on the target nucleus $T$ can be written in the compact form:

$$
\frac{1}{2 j_{\chi}+1} \frac{1}{2 j_{T}+1}|\mathcal{M}|^{2}=\frac{4 \pi}{2 j_{T}+1} \sum_{\tau=0,1} \sum_{\tau^{\prime}=0,1} \sum_{k} R_{k}^{\tau \tau^{\prime}}\left[c_{k}^{\tau},\left(v_{T}^{\perp}\right)^{2}, \frac{q^{2}}{m_{N}^{2}}\right] W_{T k}^{\tau \tau^{\prime}}(y) .
$$


In the above expression $j_{\chi}$ and $j_{T}$ are the WIMP and the target nucleus spins, respectively, $q=|\vec{q}|$ while the $R_{k}^{\tau \tau^{\prime}}$ 's are WIMP response functions (that we report for completeness in Eq.(A.1)) which depend on the couplings $c_{k}^{\tau}$ as well as the transferred momentum $\vec{q}$ and on the square of the transverse velocity in the reference frame of the center of mass of the target nucleus $\left(v_{T}^{\perp}\right)^{2}$, which can be written as:

$$
\left(v_{T}^{\perp}\right)^{2}=v_{T}^{2}-v_{\min }^{2}
$$

In particular, the quantity:

$$
v_{\min }^{2}=\frac{q^{2}}{2 \mu_{T}}=\frac{m_{T} E_{R}}{2 \mu_{T}^{2}},
$$

represents the minimal incoming WIMP speed required to impart the nuclear recoil energy $E_{R}$, while $v_{T} \equiv\left|\vec{v}_{T}\right|$ is the WIMP speed in the reference frame of the nuclear center of mass, $m_{T}$ the nuclear mass and $\mu_{T}$ the WIMP-nucleus reduced mass. In equation (2.4) the $W_{T k}^{\tau \tau^{\prime}}\left(q^{2}\right)$ 's are nuclear response functions and the index $k$ represents different effective nuclear operators, which, crucially, under the assumption that the nuclear ground state is an approximate eingenstate of $P$ and $C P$, can be at most eight: following the notation in [24], $k=M, \Phi^{\prime \prime}, \Phi^{\prime \prime} M, \tilde{\Phi}^{\prime}, \Sigma^{\prime \prime}, \Sigma^{\prime}, \Delta, \Delta \Sigma^{\prime}$. For the target nuclei $T$ used in most direct detection experiments the functions $W_{T k}^{\tau \tau^{\prime}}\left(q^{2}\right)$, calculated using nuclear shell models, have been provided in Refs.[24, 27].

In particular, the usual spin-dependent nuclear scattering process corresponds to $\mathcal{H}=$ $\sum_{\tau=0,1} c_{4}^{\tau} \mathcal{O}_{4} t^{\tau}$. The corresponding form factor is usually written in the literature in terms of the nuclear spin structure functions as[28]:

$$
S\left(q^{2}\right)=\left(c_{4}^{0}\right)^{2} S_{00}\left(q^{2}\right)+c_{4}^{0} c_{4}^{1} S_{01}\left(q^{2}\right)+\left(c_{4}^{1}\right)^{2} S_{00}\left(q^{2}\right) .
$$

At zero momentum transfer this form factor is normalized to a combination of $j_{T}$ and of the expectation values $<S_{p, n}>\equiv<T\left|S_{p, n}^{z}\right| T>$ of the projection $S^{z}$ along the $z$ axis of the total spin operators $\vec{S}_{n, p}=\sum \vec{s}_{n, p}$ of protons and neutrons on the nuclear state $\mid T>$ :

$$
S(0)=\frac{1}{\pi} \frac{\left(2 j_{T}+1\right)\left(j_{T}+1\right)}{j_{T}}\left(c_{4}^{p}<S_{p}>+c_{4}^{n}<S_{n}>\right)^{2} .
$$

In terms of the functions $W_{k}^{\tau \tau^{\prime}}$ of Ref.[24] the structure functions $S_{\tau \tau^{\prime}}$ are given by:

$$
\begin{aligned}
& S_{00}\left(q^{2}\right)=W_{\Sigma^{\prime \prime}}^{00}\left(q^{2}\right)+W_{\Sigma^{\prime}}^{00}\left(q^{2}\right) \\
& S_{11}\left(q^{2}\right)=W_{\Sigma^{\prime \prime}}^{11}\left(q^{2}\right)+W_{\Sigma^{\prime}}^{11}\left(q^{2}\right) \\
& S_{01}\left(q^{2}\right)=2\left[W_{\Sigma^{\prime \prime}}^{01}\left(q^{2}\right)+W_{\Sigma^{\prime}}^{01}\left(q^{2}\right)\right] .
\end{aligned}
$$

The above decomposition of the spin-dependent structure functions in terms of the two different non-relativistic nuclear response functions $\Sigma^{\prime \prime}$ and $\Sigma^{\prime}$ traces back to the decomposition:

$$
\vec{S}_{\chi} \cdot \vec{S}_{N}=\left(\vec{S}_{\chi} \cdot \vec{q} / q\right)\left(\vec{S}_{N} \cdot \vec{q} / q\right)+\left(\vec{S}_{\chi} \times \vec{q} / q\right) \cdot\left(\vec{S}_{N} \times \vec{q} / q\right) .
$$

In particular, $\Sigma^{\prime \prime}$ corresponds to the component of the nucleon spin along the direction of the transferred momentum $\vec{q}$ while $\Sigma^{\prime}$ to that perpendicular to it (for this reason one has $W_{\Sigma^{\prime}}^{\tau \tau^{\prime}}\left(q^{2}\right) \simeq 2 W_{\Sigma^{\prime \prime}}^{\tau \tau^{\prime}}\left(q^{2}\right)$ when $\left.q^{2} \rightarrow 0\right)$. 


\begin{tabular}{|c|c|c|c|c|c|}
\hline coupling & $R_{0 k}^{\tau \tau^{\prime}}$ & $R_{1 k}^{\tau \tau^{\prime}}$ & coupling & $R_{0 k}^{\tau \tau^{\prime}}$ & $R_{1 k}^{\tau \tau^{\prime}}$ \\
\hline 1 & $M\left(q^{0}\right)$ & - & 3 & $\Phi^{\prime \prime}\left(q^{4}\right)$ & $\Sigma^{\prime}\left(q^{2}\right)$ \\
4 & $\Sigma^{\prime \prime}\left(q^{0}\right), \Sigma^{\prime}\left(q^{0}\right)$ & - & 5 & $\Delta\left(q^{4}\right)$ & $M\left(q^{2}\right)$ \\
6 & $\Sigma^{\prime \prime}\left(q^{4}\right)$ & - & 7 & - & $\Sigma^{\prime}\left(q^{0}\right)$ \\
8 & $\Delta\left(q^{2}\right)$ & $M\left(q^{0}\right)$ & 9 & $\Sigma^{\prime}\left(q^{2}\right)$ & - \\
10 & $\Sigma^{\prime \prime}\left(q^{2}\right)$ & - & 11 & $M\left(q^{2}\right)$ & - \\
12 & $\Phi^{\prime \prime}\left(q^{2}\right), \tilde{\Phi}^{\prime}\left(q^{2}\right)$ & $\Sigma^{\prime \prime}\left(q^{0}\right), \Sigma^{\prime}\left(q^{0}\right)$ & 13 & $\tilde{\Phi}^{\prime}\left(q^{4}\right)$ & $\Sigma^{\prime \prime}\left(q^{2}\right)$ \\
14 & - & $\Sigma^{\prime}\left(q^{2}\right)$ & 15 & $\Phi^{\prime \prime}\left(q^{6}\right)$ & $\Sigma^{\prime}\left(q^{4}\right)$ \\
\hline
\end{tabular}

Table 1. Nuclear response functions corresponding to each coupling, for the velocity-independent and the velocity-dependent components parts of the WIMP response function, decomposed as in Eq.(2.11). In parenthesis the power of $q$ in the WIMP response function.

Among the $W_{k}^{\tau \tau^{\prime}}$ 's, which represent the most general nuclear response functions for WIMP-nucleus scattering, only $W_{\Sigma^{\prime \prime}}^{\tau \tau^{\prime}}$ and $W_{\Sigma^{\prime}}^{\tau \tau^{\prime}}$ have the property of being suppressed for an even number of nucleons in the target, allowing to reconcile the DAMA effect to xenon and germanium detectors by assuming $c_{i}^{n} \rightarrow 0$ (all other structure functions vanish in each different target for a different ratio $r_{i} \equiv c_{i}^{n} / c_{i}^{p}$ ). The correspondence between models and nuclear response functions can be directly read off from the WIMP response functions $R_{\Sigma^{\prime \prime}}^{\tau \tau^{\prime}}$ and $R_{\Sigma^{\prime}}^{\tau \tau^{\prime}}$ (see Eq.A.1). In particular, using the decomposition:

$$
R_{k}^{\tau \tau^{\prime}}=R_{0 k}^{\tau \tau^{\prime}}+R_{1 k}^{\tau \tau^{\prime}} \frac{\left(v_{T}^{\perp}\right)^{2}}{c^{2}}=R_{0 k}^{\tau \tau^{\prime}}+R_{1 k}^{\tau \tau^{\prime}} \frac{v_{T}^{2}-v_{\min }^{2}}{c^{2}} .
$$

this correspondence is summarized in Table 1 . We see from this table that the models which couple to the nucleus either through $W_{\Sigma^{\prime}}^{\tau \tau^{\prime}}\left(q^{2}\right)$ or $W_{\Sigma^{\prime \prime}}^{\tau \tau^{\prime}}\left(q^{2}\right)$ are $\mathcal{O}_{i}$ for $i=3,4,6,7$, 9, 10, 12, 13, 14, 15 . However, for models $\mathcal{O}_{i}$ with $i=3,12,13,15$ the spin-dependent coupling is velocity-suppressed, while the dominant velocity-independent term couples to another nuclear response function. This implies that the truly "spin-dependent" couplings are only $\mathcal{O}_{i}$ with $i=4,6,7,9,10,14$. In Table 2 , which is adopted from [24], we show which class of relativistic Effective Field theories can lead to them as a non-relativistic limit. For each relativistic model, in the last column we provide the dependence of the WIMP-nucleus scattering cross section on $q, v_{T}^{\perp}, W_{\Sigma^{\prime}}^{\tau \tau^{\prime}}\left(q^{2}\right)$ and $W_{\Sigma^{\prime \prime}}^{\tau \tau^{\prime}}\left(q^{2}\right)$.

Irrespective of their specific velocity distribution, WIMP velocities are expected to be of order $10^{-3}$, so in line 4 of Table 2 the velocity-dependent term is naturally suppressed. For this reason we have neglected it. However, notice that in line 8 the coupling term $\bar{\chi} i \sigma_{\mu \nu} \frac{q^{\nu}}{m_{M}} \gamma_{5} \chi \bar{N} \gamma_{\mu} \gamma_{5} N$ leads to a velocity-suppressed cross section $\propto\left(v_{T}^{\perp}\right)^{2}$ without the corresponding velocity-independent contribution. The same thing can be achieved by appropriate linear combinations of the operator $\bar{\chi} \gamma^{\mu} \chi \bar{N} \gamma_{\mu} \gamma^{5} N$ either with $\bar{\chi} \gamma^{\mu} \gamma_{5} \chi \bar{N} i \sigma_{\mu \nu} \frac{q^{\nu}}{m_{W I M P}} N$ or $\bar{\chi} i \sigma_{\mu \nu} \frac{q^{\nu}}{m_{W I M P}} \chi \bar{N} \gamma^{\mu} \gamma_{5} N$, as shown in lines 2 and 3 of the same Table. In Table 1 we ordered the models with powers of $q^{2}$ : actually, as we will see in the following, the scaling with $q^{2}$ will be the most relevant property to interpret our numerical results.

With the exception of line 4 and line 11, all the models of Table 2 correspond to one of the non-relativistic quantum-mechanical operators of Eq.(2.2). This also holds for the model of line 4 that can be well approximated by $\mathcal{O}_{9}$. So in the following we will refer to each of these scenarios with the corresponding $\mathcal{O}_{i}$. On the other hand, the model of line 11 is given by a superposition of $\mathcal{O}_{4}$ and $\mathcal{O}_{6}$ with both contributions of the same order. In 


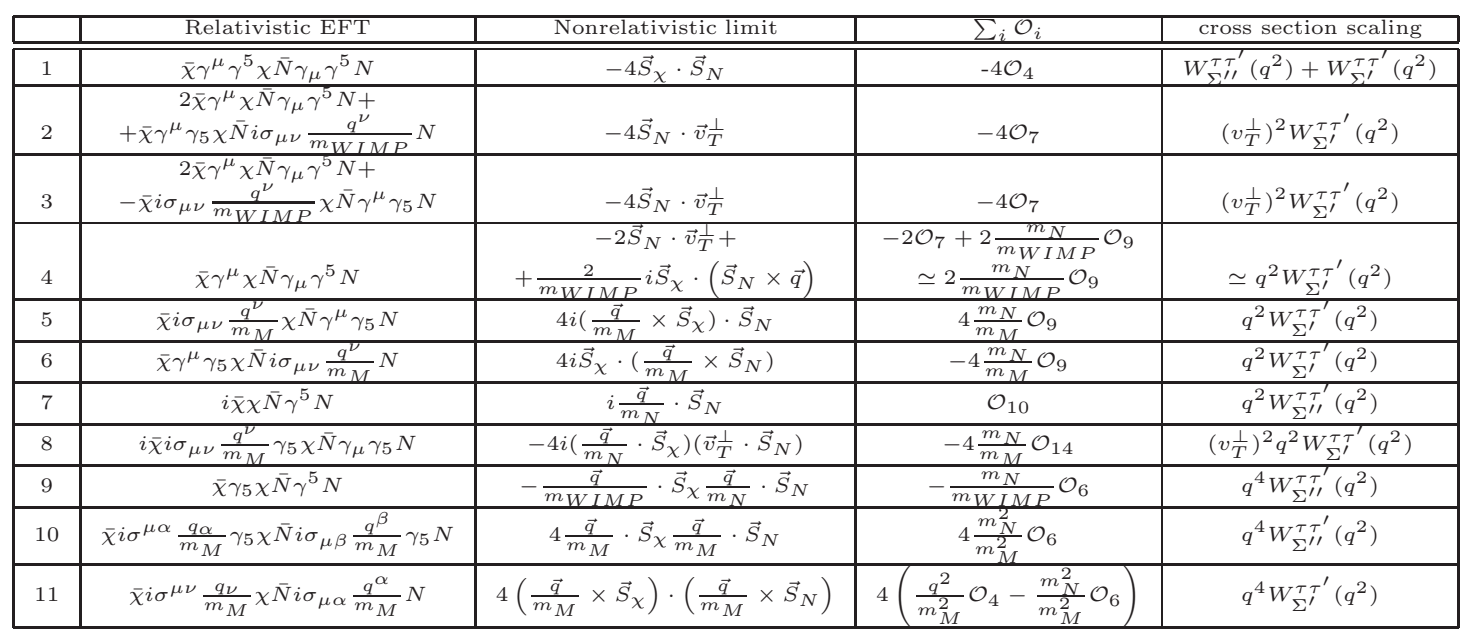

Table 2. Relativistic Effective Field Theories for a Dark Matter fermionic WIMP $\chi$ having as a low-energy limit a generalized spin-dependent $\chi$-nucleus elastic scattering. In all these scenarios the scattering cross section vanishes for all nuclei with an odd number of protons if $c_{i}^{p}=0$ and for all nuclei with an odd number of neutrons if $c_{i}^{n}=0$. Some of the interaction terms in the second column contain an arbitrary scale $m_{M}$ to ensure correct dimensionality.

Section 6 we will conventionally refer to this model as $\mathcal{O}_{46}$. In summary, we conclude that the most general spin-dependent models relevant from the point of view of the phenomenology of direct detection are seven: $\mathcal{O}_{4}, \mathcal{O}_{7}, \mathcal{O}_{9}, \mathcal{O}_{10}, \mathcal{O}_{14}, \mathcal{O}_{6}, \mathcal{O}_{46}$. We will quantitatively discuss their phenomenology in Section 6.

\section{WIMP direct detection rate}

For a given recoil energy imparted to the target the differential rate for the WIMP-nucleus scattering process is given by:

$$
\frac{d R}{d E_{R}}=\sum_{T} N_{T} \frac{\rho_{W I M P}}{m_{W I M P}} \int_{v_{\min }} d^{3} v_{T} f\left(\vec{v}_{T}\right) v_{T} \frac{d \sigma_{T}}{d E_{R}},
$$

where $\rho_{W I M P}$ is the local WIMP mass density in the neighborhood of the Sun, $N_{T}$ the number of the nuclear targets of species $T$ in the detector (the sum over $T$ applies in the case of more than one nuclear isotope), $f\left(\vec{v}_{T}\right)$ the WIMP velocity distribution, while

$$
\frac{d \sigma_{T}}{d E_{R}}=\frac{2 m_{T}}{4 \pi v_{T}^{2}}\left[\frac{1}{2 j_{\chi}+1} \frac{1}{2 j_{T}+1}\left|\mathcal{M}_{T}\right|^{2}\right]
$$

with the squared amplitude in parenthesis given explicitly in Eq.(2.4). Using Eqs (2.11,3.1,3.2) one gets:

$$
\frac{d R}{d E_{R}}=2 \sum_{T} N_{T} \frac{m_{T}}{\sigma_{r e f}} \sum_{k \tau \tau^{\prime}} W_{T k}^{\tau \tau^{\prime}}\left[y\left(E_{R}\right)\right]\left[R_{0 k}^{\tau \tau^{\prime}} \tilde{\eta}\left(v_{\min }\right)+R_{1 k}^{\tau \tau^{\prime}} \tilde{\xi}\left(v_{\min }\right)\right]
$$

where: 


$$
\begin{aligned}
& \tilde{\eta}\left(v_{\text {min }}\right)=\frac{\rho_{W I M P} \sigma_{r e f}}{m_{W I M P}} \int_{v_{\text {min }}} d^{3} \vec{v}_{T} \frac{f\left(\vec{v}_{T}\right)}{v_{T}} \\
& \tilde{\xi}\left(v_{\text {min }}\right)=\frac{\rho_{W I M P \sigma_{r e f}}}{m_{W I M P}} \int_{v_{\text {min }}} d^{3} \vec{v}_{T} \frac{f\left(\vec{v}_{T}\right)}{v_{T}}\left(v_{T}^{\perp}\right)^{2}=\int_{v_{\text {min }}} d^{3} \vec{v}_{T} \frac{f\left(\vec{v}_{T}\right)}{v_{T}}\left(v_{T}^{2}-v_{\text {min }}^{2}\right) .
\end{aligned}
$$

In Eq.(3.3) we have factorized a reference cross section $\sigma_{r e f}$ which in the case of no momentum dependence of the scattering amplitude can be identified with the large-distance total cross section for a point-like nucleus. However in the case of scattering amplitudes which vanish with $q^{2}$ the quantity $\sigma_{\text {ref }}$ is just a conventional factor with dimension -2 which must be the same for all nuclei in order to cancel out in the comparison of the expected rates in

different detectors. For all the interactions listed in Table 2 only one of the couplings $c_{k}^{p, n}$ is different from zero. In the following we choose to factorize for each case the corresponding conventional cross section $\sigma_{r e f}=\left(c_{k}^{p}\right)^{2} \mu_{\chi N}^{2} / \pi$.

Eq. (3.3) implies that, among the models summarized in Table 2, those in columns 2, 3 and 8 have a direct detection rate proportional to $\tilde{\xi}\left(v_{m i n}\right)$, while all the others scale as $\tilde{\eta}\left(v_{\min }\right)$. In both cases the same function can be factorized in all nuclei, allowing to adopt the halo-independent procedure outlined in the next Section.

\section{Halo-independent analysis}

We summarize in this Section the main formulas used in our subsequent analysis to factorize the halo-dependence from direct-detection data.

In a realistic experiment the recoil energy $E_{R}$ is obtained by measuring a related detected energy $E^{\prime}$ obtained by calibrating the detector with mono-energetic photons with known energy. However the detector response to photons can be significantly different compared to the same quantity for nuclear recoils. For a given calibrating photon energy the mean measured value of $E^{\prime}$ is usually referred to as the electron-equivalent energy $E_{e e}$ and measured in keVee. On the other hand $E_{R}$ (that represents the signal that would be measured if the same amount of energy were deposited by a nuclear recoil instead of a photon) is measured in keVnr. The two quantities are related by a quenching factor $Q$ according to $E_{e e}=Q\left(E_{R}\right) E_{R}$. Moreover the measured $E^{\prime}$ is smeared out compared to $E_{e e}$ by the energy resolution (a Gaussian smearing $\operatorname{Gauss}\left(E_{e e} \mid E^{\prime}, \sigma_{r m s}\left(E^{\prime}\right)\right) \equiv 1 /\left(\sigma_{r m s} \sqrt{2 \pi}\right) \exp \left[-\left(E^{\prime}-E_{e e}\right)^{2} /\left(2 \sigma_{r m s}^{2}\right)\right]$ with standard deviation $\sigma_{r m s}\left(E^{\prime}\right)$ related to the Full Width Half Maximum (FWHM) of the calibration peaks at $E^{\prime}$ by $F H W M=2.35 \sigma_{r m s}$ is usually assumed) and experimental count rates depend also on the counting efficiency or cut acceptance $\epsilon\left(E^{\prime}\right)$. Overall, the expected differential event rate is given by:

$$
\frac{d R}{d E^{\prime}}=\epsilon\left(E^{\prime}\right) \int_{0}^{\infty} d E_{e e} \operatorname{Gauss}\left(E_{e e} \mid E^{\prime}, \sigma_{r m s}\left(E^{\prime}\right)\right) \frac{1}{Q\left(E_{R}\right)} \frac{d R}{d E_{R}}
$$

with $d R / d E_{R}$ given by Eq.(3.1). Then the number of events in a given interval of the detected energy $E^{\prime}$ is simply:

$$
R_{\left[E_{1}^{\prime}, E_{2}^{\prime}\right]}=\int_{E_{1}^{\prime}}^{E_{2}^{\prime}} \frac{d R}{d E^{\prime}} .
$$


Combining Eqs.(4.1,4.2) with Eq. (3.3) and changing variable from $E_{R}$ to $v_{\min }$ the expected rate can be cast in the compact form:

$$
R_{\left[E_{1}^{\prime}, E_{2}^{\prime}\right]}=\frac{\rho_{W I M P}}{m_{W I M P}} \int_{0}^{\infty} d v_{\min } \tilde{\eta}\left(v_{\min }\right) \mathcal{R}_{\left[E_{1}^{\prime}, E_{2}^{\prime}\right]}^{0}\left(v_{\min }\right),
$$

for models without velocity suppression and:

$$
R_{\left[E_{1}^{\prime}, E_{2}^{\prime}\right]}=\frac{\rho_{W I M P}}{m_{W I M P}} \int_{0}^{\infty} d v_{\min } \tilde{\xi}\left(v_{\min }\right) \mathcal{R}_{\left[E_{1}^{\prime}, E_{2}^{\prime}\right]}^{1}\left(v_{\min }\right),
$$

for those with velocity suppression, with:

$$
\begin{aligned}
\mathcal{R}_{\left[E_{1}^{\prime}, E_{2}^{\prime}\right] \equiv}^{\equiv} & \int_{0}^{\infty} d v_{\min } \sum_{T} 8 N_{T} \mu_{T}^{2} \frac{1}{\sigma_{r e f}} \sum_{k} \sum_{\tau \tau^{\prime}} R_{m k}^{\tau \tau^{\prime}}\left[q^{2}\left(v_{\min }\right)\right] \frac{W_{T k}^{\tau \tau^{\prime}}\left[y\left(v_{\min }\right)\right]}{2 j_{T}+1} \frac{1}{Q\left[E_{R}\left(v_{\min }\right)\right]} \times \\
& \int_{E_{1}^{\prime}}^{E_{2}^{\prime}} d E^{\prime} \epsilon\left(E^{\prime}\right) \operatorname{Gauss}\left(E_{e e}\left[E_{R}\left(v_{\min }\right)\right] \mid E^{\prime}, \sigma_{r m s}\left(E^{\prime}\right)\right), \quad m=0,1 .
\end{aligned}
$$

In the present analysis we will assume that the yearly modulation effect observed by the DAMA experiment is due to the time-dependence of $\tilde{\eta}$ or $\tilde{\xi}$ due to the rotation of the Earth around the Sun. So we also introduce the modulated amplitudes:

$$
\begin{aligned}
& \tilde{\eta}_{1}\left(v_{\min }\right) \equiv \tilde{\eta}\left(v_{\min }, t=t_{\max }\right)-\tilde{\eta}\left(v_{\min }, t=t_{\min }\right), \\
& \tilde{\xi}_{1}\left(v_{\min }\right) \equiv \tilde{\xi}\left(v_{\min }, t=t_{\max }\right)-\tilde{\xi}\left(v_{\min }, t=t_{\min }\right),
\end{aligned}
$$

with $t_{\max }$ and $t_{\min }$ the times of the year corresponding to the maximum and to the minimum of the signal. The modulated amplitudes measured by DAMA that we will use in Section 5 to get estimations of $\tilde{\eta}_{1}$ and $\tilde{\xi}_{1}$ were obtained by fitting the data with a time dependence parametrized with the functional form:

$$
R_{\left[E_{1}^{\prime}, E_{2}^{\prime}\right]}=R_{\left[E_{1}^{\prime}, E_{2}^{\prime}\right]}^{0}+R_{\left[E_{1}^{\prime}, E_{2}^{\prime}\right]}^{1} \cos \left[\omega\left(t-t_{\max }\right)\right],
$$

which is expected when the isothermal sphere model is adopted for the WIMP speed distribution. Moreover, according to the DAMA analysis, the modulation phase is in (rough) agreement to what is expected for the isothermal sphere model, i.e. $t_{\max } \simeq 2 \mathrm{June}$ (and, consequently, $t_{\min } \simeq 2$ December). However, a truly halo independent analysis would require to reanalyze the experimental data allowing for functional forms of general nature beyond Eq. (4.7 $)^{2}$. Nevertheless, in the following to estimate the $\tilde{\eta}_{1}, \tilde{\xi}_{1}$ functions we will make directly use of the published DAMA modulation amplitudes, implicitly assuming that indeed those estimations do not differ significantly from what one would obtain using a more general form to analyze the data. With this important proviso, in the present analysis we wish to make the smallest possible number of assumptions on the functions $\tilde{\eta}, \tilde{\xi}$. They turn out to be the same, and in the case of $\tilde{\eta}$ they reduce to:

\footnotetext{
${ }^{2}$ For speed distributions beyond the Isothermal Sphere the time dependence may differ from that of Eq.(4.7). For instance, it has been shown that in anisotropic extensions of the isothermal sphere the phase and even the period may vary[29].
} 


$$
\begin{array}{cl}
\tilde{\eta}\left(v_{\min , 2}\right) \leq \tilde{\eta}\left(v_{\min , 1}\right) & \text { if } v_{\min , 2}>v_{\min , 1} \\
\tilde{\eta}_{1} \leq \tilde{\eta} & \text { at the same } v_{\min } \\
\tilde{\eta}\left(v_{\min } \geq v_{e s c}\right)=0 . &
\end{array}
$$

The first condition descends from the definitions (3.4), that implies that both $\tilde{\eta}\left(v_{\min }\right)$ and $\tilde{\xi}\left(v_{\min }\right)$ are decreasing functions of $v_{\min }$. The second is an obvious consequence of the fact that $\tilde{\eta}_{1}, \tilde{\xi}_{1}$ are the modulated parts of $\tilde{\eta}$ and $\tilde{\xi}$. The last condition reflects the requirement that the WIMPs are gravitationally bound to our Galaxy. In the following we will assume that the WIMP halo is at rest in the Galactic rest frame and we will adopt as the maximal velocity of WIMPs $v_{e s c}=782 \mathrm{~km} / \mathrm{s}$ in the reference frame of the laboratory, by combining the reference value of the escape velocity $v_{\text {esc }}^{\text {Galaxy }}=550 \mathrm{~km} / \mathrm{s}$ in the galactic rest frame with the velocity $v_{0}=232 \mathrm{~km} / \mathrm{s}$ of the Solar system with respect to the WIMP halo.

So, given an experiment with detected count rate $N_{\text {exp }}$ in the energy interval $E_{1}^{\prime}<E^{\prime}<$ $E_{2}^{\prime}$ the combination:

$$
<\tilde{\eta}>=\frac{\int_{0}^{\infty} d v_{v \min } \tilde{\eta}\left(v_{\min }\right) \mathcal{R}_{\left[E_{1}^{\prime}, E_{2}^{\prime}\right]}\left(v_{\min }\right)}{\int_{0}^{\infty} d v_{\min } \mathcal{R}_{\left[E_{1}^{\prime}, E_{2}^{\prime}\right]}\left(v_{\min }\right)}=\frac{N_{\exp }}{\int_{0}^{\infty} d v_{\min } \mathcal{R}_{\left[E_{1}^{\prime}, E_{2}^{\prime}\right]}\left(v_{\min }\right)},
$$

can be interpreted as an average of the function $\tilde{\eta}\left(v_{\min }\right)$ in an interval $v_{\min , 1}<v_{\min }<v_{\min , 2}$, with an analogous definition for the average of the function $\tilde{\xi}\left(v_{\min }\right)$. The $v_{\min }$ interval is defined as the one where the response function $\mathcal{R}$ is "sizeably" different from zero (we will conventionally take the interval $v_{\min }\left[E_{R}\left(E_{e e, 1}\right)\right]<v_{\min }<v_{\min }\left[E_{R}\left(E_{e e, 2}\right)\right]$ with $E_{e e, 1}=$ $E_{1}^{\prime}-\sigma_{r m s}\left(E_{1}^{\prime}\right), E_{e e, 2}=E_{2}^{\prime}+\sigma_{r m s}\left(E_{2}^{\prime}\right)$, i.e. the $E^{\prime}$ interval enlarged by the energy resolution).

\section{Constraints from direct detection experiments and compatibility factor}

In this Section we will give explicit formulas for the case of a velocity-independent cross section where the expected rate is given by Eq.(4.3) and depends on $\tilde{\eta}\left(v_{\min }\right)$. Clearly, the velocity-dependent case of Eq.(4.4) with dependence on $\tilde{\xi}\left(v_{\min }\right)$ is analogous.

Following the halo-independent procedure outlined in Section 4 it is straightforward, for a given choice of the DM parameters, to obtain estimations $\overline{\tilde{\eta}}_{1, i}^{D A M A-N a}$ of the modulationamplitude function $\tilde{\eta}_{1}\left(v_{\text {min }}\right)$ averaged in appropriately chosen $v_{m i n, i}$ intervals mapped from the DAMA experimental annual modulation amplitudes. Using the condition $\tilde{\eta}_{1}\left(v_{\text {min }}\right) \leq$ $\tilde{\eta}\left(v_{\min }\right)$ this allows to get lower bounds on the $\tilde{\eta}\left(v_{\text {min }}\right)$ function, which can be compared to upper bounds $\overline{\tilde{\eta}}_{j, \text { lim }}$ on the same quantity derived from the data of the experiments that have reported null results. Among the latter, the KIMS experiment uses iodine, one of the same targets of DAMA ( $\mathrm{NaI}$ for DAMA vs. $\mathrm{CsI}$ for KIMS). KIMS has reported an upper bound on the unmodulated WIMP rate in the low-part of its measured spectrum that is about a factor of two smaller than the modulated amplitude measured by DAMA in the same energy interval[6]. This implies that, as far as the WIMP rate in both experiments is dominated by scatterings on iodine, the two results cannot be reconciled by changing either the halo function or the scaling law of the cross section. In this case the only way to reconcile the two conflicting results is to look deeper in the possible sources of systematic errors, including the many uncertainties connected to quenching factors, atomic form factors, background cuts efficiencies, etc. Moreover, as already pointed out, as far as DAMA is concerned the halofunction factorization is only possible if WIMPs scatter predominantly on a single target 
nucleus. For both reasons in the following we will restrict our analysis to a range of the WIMP mass for which the DAMA effect can be consistently explained with scatterings on sodium targets only. In the popular case of an Isothermal Sphere for the velocity distribution this is achieved for $m_{W I M P} \lesssim 20 \mathrm{GeV}$. However this range for $m_{W I M P}$ can be larger if the WIMP velocity distribution is different from a Maxwellian and if the only assumptions on the halo function are those of Eq.(4.8). In particular it can be maximally extended by assuming that the function $\tilde{\eta}$ is the minimal one compatible to the DAMA signal. Assuming that the latter corresponds for sodium to the $v_{\min }$ range $v_{1}^{D A M A, N a} \leq v_{\min } \leq v_{2}^{D A M A, N a}$ and for iodine to the range $v_{1}^{D A M A, I} \leq v_{\min } \leq v_{2}^{D A M A, I}$, the most conservative assumption on $\tilde{\eta}$ is that it vanishes for $v_{\min }>v_{2}^{D A M A, N a}$ (in particular, this is consistent with the minimal requirement that the halo function $\eta$ is a decreasing function $v_{\text {min }}$.). Then, as long as $v_{1}^{D A M A, I}>v_{2}^{D A M A, N a}$, one can conservatively assume that scatterings on iodine are below the energy threshold of DAMA and do not contribute to the modulation effect. Using Eq.(2.6) this corresponds to $m_{W I M P} \lesssim 60 \mathrm{GeV}$, assuming the energy range 2 keVee $\leq E^{\prime} \leq$ $4 \mathrm{keVee}$ for the DAMA modulation region, enlarged by an energy resolution $\simeq 0.448 \times \sqrt{E^{\prime}}$ keVee [2]. In the same range of $m_{W I M P}$ also scatterings on iodine and caesium are below the threshold of 3 keVee in KIMS (assuming the latest measurement of the KIMS quenching factor [15], which is similar to that of DAMA). As a consequence of this in the following we will restrict our analysis to $m_{W I M P} \lesssim 60 \mathrm{GeV}$, assume only scatterings on sodium in DAMA and neglect the KIMS constraint.

Quantitatively, for a given choice of the WIMP mass $m_{W I M P}$, of the interaction term from Table 2 and of the corresponding ratio $r_{i}$, the compatibility between DAMA and all the other results can be assessed introducing the following compatibility ratio[26]:

$$
\mathcal{D}\left(m_{W I M P}, r_{i}\right) \equiv \max _{i \in \operatorname{signal}}\left(\frac{\overline{\tilde{\eta}}_{i}^{D A M A-N a}+\sigma_{i}}{\min _{j \leq i} \overline{\tilde{\eta}}_{j, l i m}}\right),
$$

where $\sigma_{i}$ represents the standard deviation on $\overline{\tilde{\eta}}_{i}^{D A M A-N a}$ as estimated from the data, $i \in$ signal means that the maximum of the ratio in parenthesis is for $v_{m i n, i}$ corresponding to the DAMA excess, while, due to the fact that the function $\tilde{\eta}$ is non-decreasing in all velocity bins $v_{\text {min }, i}$, the denominator contains the most constraining bound on $\tilde{\eta}$ for $v_{\min , j} \leq v_{\min , i}$. The latter minimum includes all available bounds from scintillators, ionizators and calorimeters (see Appendix B for a summary of the experimental inputs used in our analysis). Specifically, compatibility between DAMA and the constraints included in the calculation of Eq.(5.1) is ensured if $\mathcal{D}<1$. Notice that, by combining different $v_{m i n, i}$ bins, the above definition takes into account the most general momentum dependencies predicted by the various effective interaction terms of Table 2.

The procedure outlined above cannot include bubble chambers and droplet detectors which are only sensitive to the energy threshold, such as SIMPLE[11](using $\mathrm{C}_{2} \mathrm{ClF}_{5}$ ), COUPP [12] (using $C F_{3} I$ ), PICASSO[13] (using $C_{4} F_{10}$ ) or PICO-2L[14] (using $C_{3} F_{8}$ ), since in this case it is not possible to map the corresponding bounds to arbitrary velocity bins. Notice that these experiments contain all proton-odd elements (fluorine, chlorine and iodine), so they are particularly relevant for our analysis since they are complementary to germanium and xenon detectors which are neutron-odd. Moreover, another complication in using the compatibility factor defined in Eq.(5.1) to assess agreement between the DAMA excess and this class of experiments is that they all contain different nuclear targets, so that it is in general not possible to factorize the $\tilde{\eta}$ function and it is not trivial (as for instance 
for the case of a coherent interaction, where the cross section scales with the square of the atomic mass number) to single out particular kinematic situations where one of the targets dominates, this also because of the momentum-dependence of some of the interactions. So, in order to impose the bounds from this class of experiments in a halo-independent way, we need to generalize the compatibility factor definition of Eq.(5.1). In order to do so, we proceed in the following way: i) we use the experimental DAMA modulation-amplitudes to get a conservative piecewise estimation $\tilde{\eta}_{1}^{\text {est }}\left(v_{\min }\right)$ of the minimal $\tilde{\eta}_{1}$ modulated halo function compatible to the signal; ii) we obtain the corresponding estimation of the unmodulated part $\tilde{\eta}^{e s t}\left(v_{\text {min }}\right)$ by requiring that it is a decreasing function of $v_{\text {min }}$ with $\tilde{\eta}^{\text {est }}\left(v_{\text {min }}\right) \geq \tilde{\eta}_{1}^{\text {est }}\left(v_{\text {min }}\right)$; iii) in compliance with (4.8) and with the goal of obtaining a conservative bound, we require that the function $\tilde{\eta}$ is the minimal one able to explain the DAMA effect, so we assume

$\tilde{\eta}^{e s t}\left(v_{\min }>v_{2}^{D A M A, N a}\right)=0$ (this is also consistent to dominance of scatterings on sodium in DAMA in the mass range $m_{W I M P}<60 \mathrm{GeV}$, as explained above); iv) we then use $\tilde{\eta}^{e s t}\left(v_{\text {min }}\right)$ to directly calculate for each experiment among $k=$ SIMPLE, COUPP, PICASSO and PICO$2 \mathrm{~L}$ and for each energy threshold $E_{t h, i}$ the expected number of WIMP events $N_{k, i}^{\text {expected }}$ and compare it to the corresponding upper bound $N_{k, i}^{\text {bound }}$ (see Appendix B for further details). Then, a straightforward generalization of the compatibility factor of Eq.(5.1) is:

$$
\mathcal{D}\left(m_{W I M P}, r_{i}\right) \rightarrow \max \left(\mathcal{D}\left(m_{W I M P}, r_{i}\right), \frac{N_{k, i}^{\text {expected }}}{N_{k, i}^{\text {bound }}}\right) .
$$

Notice that the above procedure is very general, since it allows to get a halo-independent bound in the case of any experiment that does not observe a signal. In particular this means that the requirement that one target dominates the expected rate is only needed to get estimations of the halo function from those experiments that observe an excess, but is not indispensable to get a conservative bound for those reporting a null result. In the following Section we will use the above definition of the compatibility factor to explore the WIMP parameter space for the generalized spin-dependent interactions introduced in Section 2.

\section{Results}

Each of the seven phenomenological models $\mathcal{O}_{i}$ (with $i=4,6,7,9,10,14,46$ ) listed at the end of Section 2 depends on three parameters: the WIMP mass $m_{W I M P}$ and the two components $\left(c_{i}^{p}, c_{i}^{n}\right)$ of the corresponding effective coupling. However, following the halo-independent approach summarized in Section 4, we get our estimations of the $\tilde{\eta}$ or $\tilde{\xi}$ functions factorizing out the reference cross section $\sigma_{r e f}=c_{i}^{p} \mu_{\chi N} / \pi$. As a consequence of this the coupling $c_{i}^{p}$ cancels out in our definition $(5.1,5.2)$ of the compatibility factor $\mathcal{D}$. So in each model the phenomenology depends only on two parameters: $m_{W I M P}$ and the ratio $r_{i}$ (in the case of model $\mathcal{O}_{46}$ we factorize $\left.\sigma_{r e f}=c_{6}^{p} \mu_{\chi N} / \pi\right)$.

Moreover, the pairs of models $\mathcal{O}_{4}-\mathcal{O}_{7}$ and $\mathcal{O}_{9}-\mathcal{O}_{14}$ only differ for an additional $\left(v_{T}^{\perp}\right)^{2}$ factor in the cross section (see Table 2), which is absorbed in the definition of the halo function $\tilde{\xi}$. So in each pair the two models have identical compatibility factors $\mathcal{D}$ (in each case the estimations $\tilde{\xi}_{i}$ for the velocity-dependent model are simply rescaled by a common factor with respect to the corresponding $\tilde{\eta}_{i}$ in the velocity-independent one) . The bottom line is that only five relevant cases remain: $\mathcal{O}_{4}, \mathcal{O}_{6}, \mathcal{O}_{9}, \mathcal{O}_{10}$ and $\mathcal{O}_{46}$.

The results of our analysis is shown in Fig.1. The left-hand panel shows the contour plot in the $m_{W I M P}-r_{i}$ plane for $\mathcal{D}=1$ of models $\mathcal{O}_{i}, i=6,46,9,10$ and $\mathcal{D}=1.7$ for $\mathcal{O}_{4}$; the 

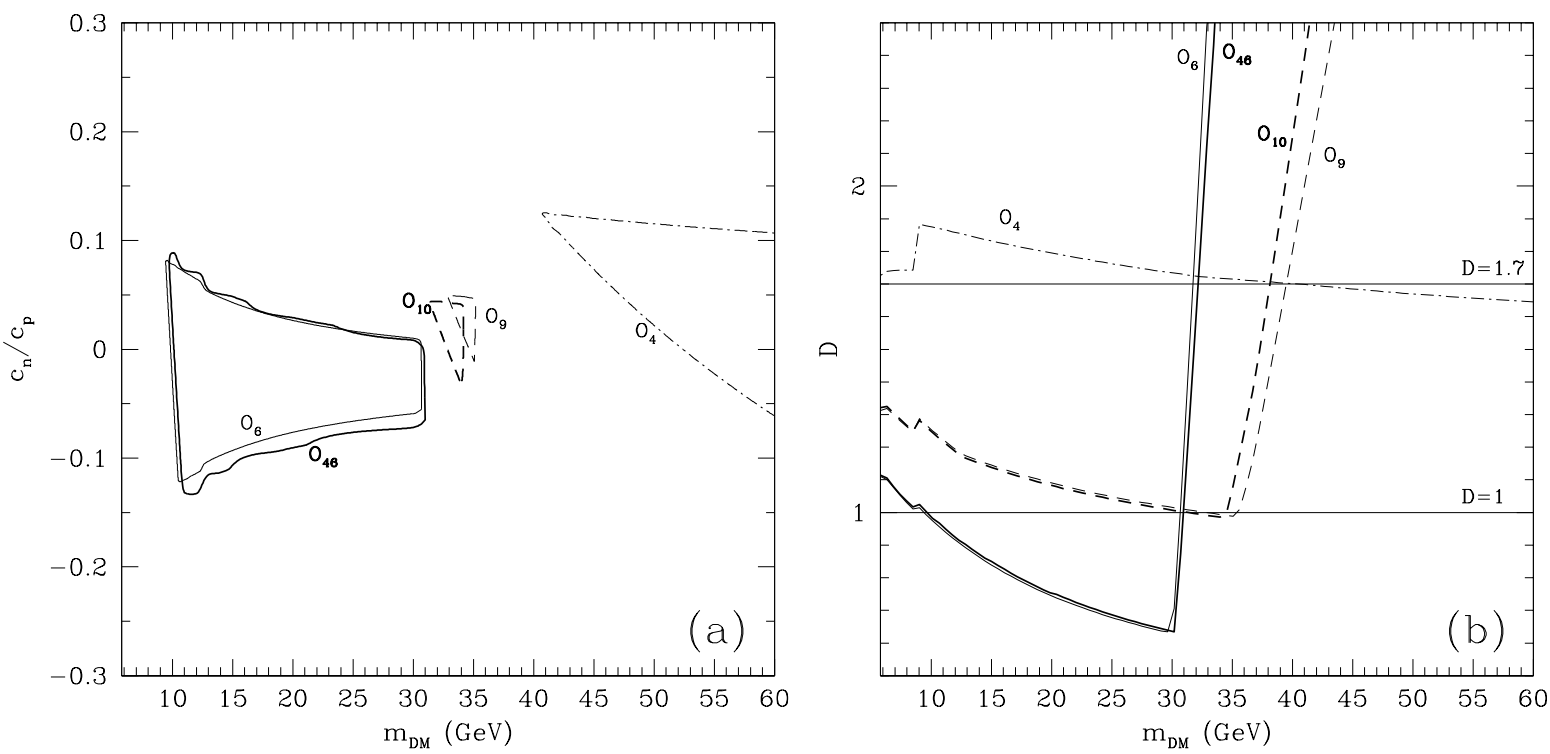

Figure 1. (a) Contour plot in the $m_{W I M P}-c_{i}^{n} / c_{i}^{p}$ plane for the compatibility factor $\mathcal{D}$ defined in Eqs. (5.1,5.2). The constant value $\mathcal{D}=1$ is shown for models $\mathcal{O}_{i}, i=6,46,9,10$, while $\mathcal{D}=1.7$ is plotted for $\mathcal{O}_{4}$. (b) For the same models $\mathcal{O}_{i}$ the minimum of $\mathcal{D}$ is plotted as a function of $m_{W I M P}$ when $c_{i}^{n} / c_{i}^{p}$ is marginalized.

right-hand panel shows the minimum of $\mathcal{D}$ as a function of $m_{W I M P}$ when $r_{i}$ is marginalized. A few features arise from both plots: i) when $c^{n} / c^{p} \rightarrow 0$ some intervals of $m_{W I M P}$ exist where the DAMA effect is compatible to constraints from other direct-detection experiments for generalized spin-dependent interactions $\left(\mathcal{O}_{i}, i=6,46,9,10\right)$, while the compatibility factor for the standard spin-dependent interaction $\mathcal{O}_{4}$ is always above $\mathcal{D}=1$; ii) from Table 2 one can see that the pairs of models $\mathcal{O}_{6}-\mathcal{O}_{46}$ and $\mathcal{O}_{9}-\mathcal{O}_{10}$ have the same momentum dependence, while they differ for the nuclear response function: since however $W_{\Sigma^{\prime}}\left(q^{2}\right) \simeq 2 W_{\Sigma^{\prime \prime}}\left(q^{2}\right)$ (at least for small $q^{2}$ ) the compatibility factor $\mathcal{D}$ has very similar behaviours for the models in each pair ; ii) the tension between DAMA and other experiments is better alleviated in those models where momentum dependence is largest: in fact, models $\mathcal{O}_{6}$ and $\mathcal{O}_{46}$, which reach the best compatibility, depend on momentum through a factor $q^{4}$ compared to $\mathcal{O}_{9}$ and $\mathcal{O}_{10}$ where the dependence is through $q^{2}$ (see again Table 2 ); iv) for $m_{W I M P} \gtrsim 30 \mathrm{GeV}$ the compatibility factor rises steeply for models $\mathcal{O}_{6}, \mathcal{O}_{46}, \mathcal{O}_{9}$ and $\mathcal{O}_{10}$, while no such feature is observed for the case $\mathcal{O}_{4}$.

The last two properties can be understood in the following way. If $m_{W I M P} \lesssim 30 \mathrm{GeV}$, the WIMP signal in bubble chambers and droplet detectors is dominated by scatterings off fluorine. In particular, in this $m_{W I M P}$ range in order to deposit recoil energies above threshold scatterings off iodine in COUPP require $v_{\min }$ values beyond the corresponding range for the DAMA signal, and we make the conservative assumption that in this case the halo function $\tilde{\eta}$ vanishes. Moreover scatterings off chlorine in SIMPLE are subdominant due to the suppressed nuclear response function. In this case the transferred momenta $q^{2}$ which explain the DAMA modulation effect for WIMP scatterings off sodium in DAMA are larger than the corresponding ones off fluorine in COUPP, PICASSO and PICO-2L. For instance, for $m_{W I M P}=25 \mathrm{GeV}$ one has $285 \mathrm{MeV}^{2} \lesssim\left(q_{D A M A}^{N a}\right)^{2} \lesssim 570 \mathrm{MeV}^{2}, 275 \mathrm{MeV}^{2} \lesssim\left(q_{\text {COUPP }}^{F}\right)^{2} \lesssim 470$ 

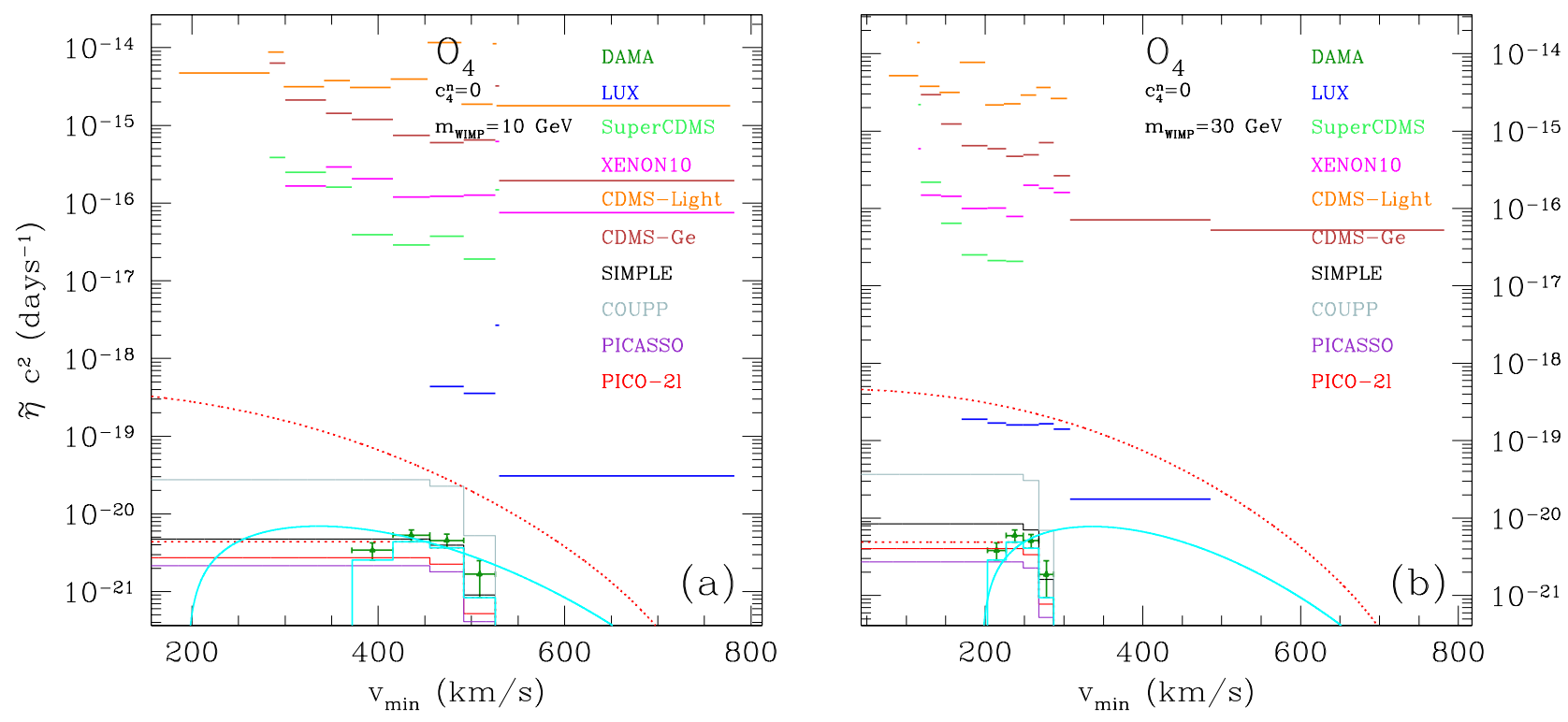

Figure 2. Measurements and bounds for the function $\tilde{\eta}$ defined in Eq. (3.4) for model $\mathcal{O}_{4}$ (standard spin-dependent interaction) and $c_{4}^{n}=0$. (a) $m_{W I M P}=10 \mathrm{GeV}$; (b) $m_{W I M P}=30 \mathrm{GeV}$. The $\tilde{\xi}$ determinations for model $\mathcal{O}_{7}$ would be rescaled by an approximately common factor $\simeq 3 / 2$ with respect to the $\tilde{\eta}$ values shown in this figure.

$\mathrm{MeV}^{2}, 60 \mathrm{MeV}^{2} \lesssim\left(q_{\text {PICASSO }}^{F}\right)^{2} \lesssim 470 \mathrm{MeV}^{2}, 113 \mathrm{MeV}^{2} \lesssim\left(q_{\text {PICO-2L }}^{F}\right)^{2} \lesssim 470 \mathrm{MeV}^{2}$. This implies that models where the expected detection rate depends on one additional factor $\left(q^{2}\right)^{n}, n>0$ such as $\mathcal{O}_{i}, i=6,46,9,10$ present a relative enhancement of the expected rate in DAMA compared to that in fluorine detectors, with a consequent relative loss of sensitivity for the latter. An exception to this argument is SIMPLE, where the $q^{2}$ interval for scatterings off fluorine has more overlap with that of DAMA, $283 \mathrm{MeV}^{2}<q_{S I M P L E}^{F}<470 \mathrm{MeV}^{2}$, but which is overall less constraining than the other detectors due to the lower exposure (see Appendix B). On the other hand, for $m_{W I M P} \gtrsim 30 \mathrm{GeV}$ scatterings off iodine in COUPP become kinematically allowed, with values of the transferred momenta which are much larger than those related to fluorine and sodium. For instance, for $m_{W I M P}=35 \mathrm{GeV}$ one has 1850 $\mathrm{MeV}^{2} \lesssim\left(q_{\text {COUPP }}^{I}\right)^{2} \lesssim 2350 \mathrm{MeV}^{2}$. Clearly, this implies a strong enhancement of the expected signal in COUPP for interactions involving an additional dependence on $q^{2}$, with a consequent steep rise of the compatibility factor $\mathcal{D}$, as observed in Fig. 1. This effect is not present for the standard spin-dependent interaction $\mathcal{O}_{4}$, whose expected rate has no $q^{2}$ dependence.

In order to discuss in more detail the phenomenology in Figures 2, 3 and 4 the measurements and bounds for the function $\tilde{\eta}$ obtained using Eq. (4.9) are plotted as a function of $v_{\text {min }}$. In particular, in all these figures we take $r_{i}=0$. Fig. 2(a) shows the case of a standard spin-dependent interaction $\mathcal{O}_{4}$ when $m_{W I M P}=10 \mathrm{GeV}$, while Fig. 2(b) shows the same when $m_{W I M P}=30 \mathrm{GeV}$. On the other hand, Figure $3(\mathrm{a})$ shows the case of model $\mathcal{O}_{6}$ for $m_{W I M P}=10 \mathrm{GeV}$ while Fig. $3(\mathrm{~b})$ shows the same when $m_{W I M P}=30 \mathrm{GeV}$. Finally, in Figure 2 (a) and $2(\mathrm{~b})$ model $\mathcal{O}_{9}$ is shown for $m_{W I M P}=10 \mathrm{GeV}$ and $m_{W I M P}=33 \mathrm{GeV}$, respectively.

As discussed before, phenomenology of these models is practically degenerate with other scenarios. In particular, the $\tilde{\xi}$ determinations for model $\mathcal{O}_{7}$ would be rescaled by an approximately common factor $(\simeq 3 / 2)$ with respect to the $\tilde{\eta}$ shown in figure 2 for $\mathcal{O}_{4}$. In the same 
way the phenomenology of $\mathcal{O}_{46}$ is very similar to model $\mathcal{O}_{6}$, apart from an overall approximate factor $(\simeq 1 / 2)$ in the $\tilde{\eta}$ determinations, so it can be described by Figure 3. Moreover, the $\tilde{\xi}$ determinations for model $\mathcal{O}_{14}$ would be rescaled by an exact common factor of 2 with respect to the $\tilde{\eta}$ values shown in Figure 4 for $\mathcal{O}_{9}$; on the other hand, the $\tilde{\eta}$ determinations for model $\mathcal{O}_{10}$ would be rescaled by an approximately common factor $\simeq 1 / 2$.

From all these plots we observe that germanium detectors and LUX imply relatively lax constraints, due to the suppression of their nuclear response functions when $c_{i}^{n}=0$ (and, in the case of germanium, for the small natural abundance of ${ }^{73} \mathrm{Ge}$, the only isotope carrying spin).

Moreover, in each plot the step-like cyan curve represents our conservative estimation $\tilde{\eta}_{1}^{e s t}\left(v_{\text {min }}\right)$ of the minimal $\tilde{\eta}_{1}$ modulated halo function compatible to the DAMA signal. In particular, as explained before, we assume that $\tilde{\eta}_{1}^{e s t}\left(v_{\min }\right)$ vanishes outside the DAMA $v_{\text {min }}$ interval. The corresponding estimation $\tilde{\eta}^{e s t}\left(v_{\text {min }}\right) \geq \tilde{\eta}_{1}^{e s t}\left(v_{\text {min }}\right)$ of the minimal $\tilde{\eta}$ function is obtained by requiring it to be a decreasing function of $v_{\min }$ and is represented by the piecewise dotted red line.

As explained before, we use the function $\tilde{\eta}^{\text {est }}\left(v_{\min }\right)$ to calculate the expected rate on droplet detectors and bubble chambers in the compatibility factor of Eq.(5.2). We decide to show the corresponding constraints by plotting for each experiment the function $\tilde{\eta}^{e s t}\left(v_{\text {min }}\right)$ rescaled by a constant factor in such a way that the corresponding expected rate is equal to the corresponding most constraining upper bound on the number of events. As can be seen from all the figures, also in the most favorable situations $\left(\mathcal{O}_{6} \simeq \mathcal{O}_{46}\right.$ for $\left.m_{W I M P} \simeq 30 \mathrm{GeV}\right)$ the bound is always relatively close (within one sigma) to the minimal DAMA signal. Since the DAMA estimations are for $\tilde{\eta}_{1}$ while the bounds are for $\tilde{\eta}$ this implies that compatibility among them necessarily requires a modulation amplitude fraction in DAMA much larger then that predicted for a standard Isothermal Sphere (which is typically below 10\%). In the same figures the continuous cyan line and dotted red line represent $\tilde{\eta}_{1}^{\text {Maxwellian }}\left(v_{\text {min }}\right)$ and

$\tilde{\eta}^{\text {Maxwellian }}\left(v_{\text {min }}\right)$ for the case of a Maxwellian velocity distribution, where $\tilde{\eta}_{1}^{\text {Maxwellian }}\left(v_{\text {min }}\right)$ is normalized in such a way to minimize a $\chi$-square with the DAMA modulation amplitudes. Indeed, the red dotted line is well above existing constraints on $\tilde{\eta}$ in all cases. Moreover, notice that a Maxwellian for $m_{W I M P}=30 \mathrm{GeV}$ predicts a sizeable contribution to the event rate due to scatterings off iodine, so this explain the particularly poor fit with the $\tilde{\eta}$ estimations for DAMA, where dominance on sodium was assumed.

\section{Conclusions}

In the present paper we have used non-relativistic Effective Field Theory to classify the most general spin-dependent WIMP-nucleus interactions, and within this class of models we have discussed the viability of an interpretation of the DAMA modulation result in terms of a WIMP signal, using a halo-independent approach in which all dependencies from astrophysics are factorized in a single halo function.

One of the main motivations of the spin-dependent scenario is the fact that the most stringent bounds on the interpretation of the DAMA effect in terms of WIMP-nuclei scatterings arise today from detectors using xenon (XENON100, LUX) and germanium (CDMS) whose spin is mostly originated by an unpaired neutron, while both sodium and iodine in DAMA have an unpaired proton: if the WIMP effective coupling to neutrons is suppressed compared to that on protons this class of bounds can be evaded. In this case the most constraining remaining bounds arise from droplet detectors (SIMPLE, COUPP) and bubble 

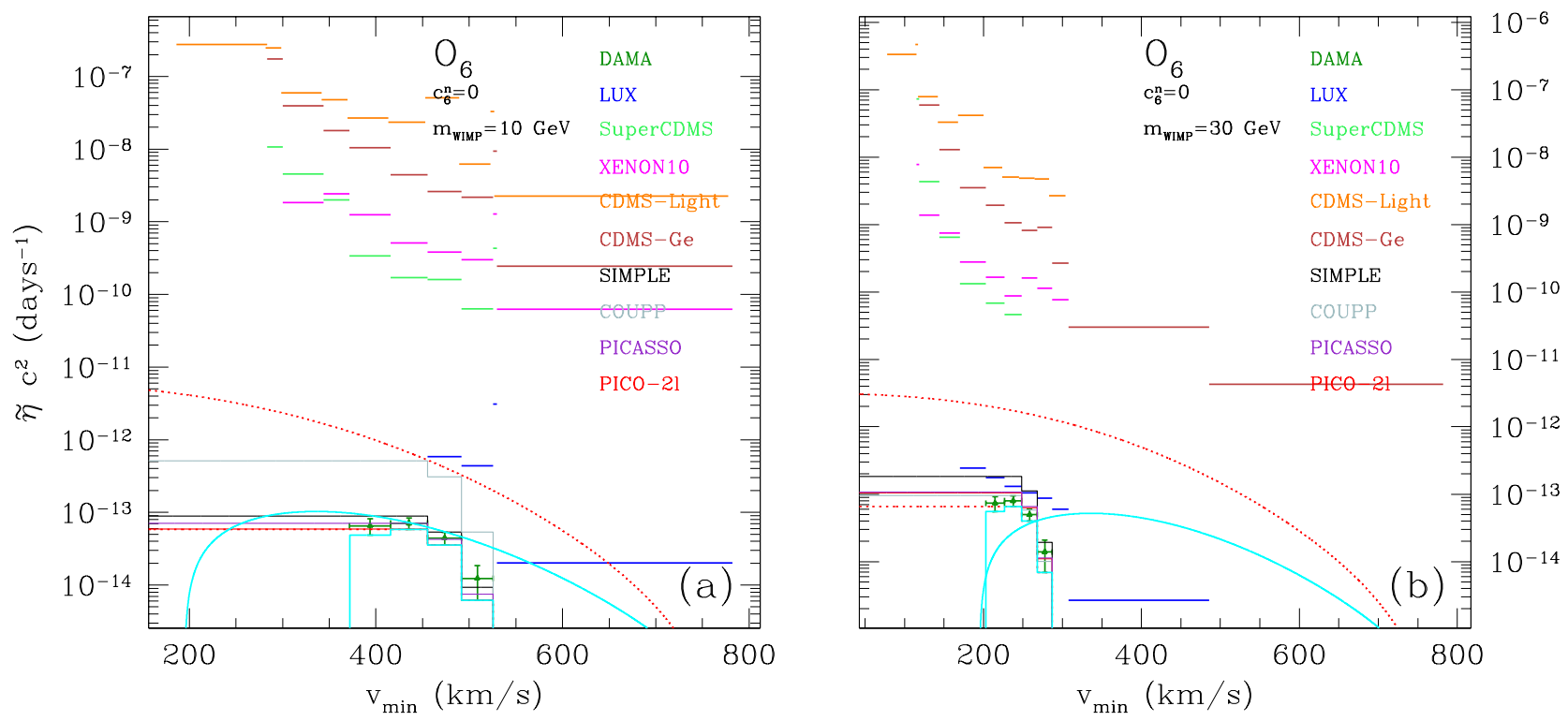

Figure 3. The same as in Figure 2 for model $\mathcal{O}_{6}$. The $\tilde{\eta}$ determinations for model $\mathcal{O}_{46}$ would be rescaled by an approximately common factor $\simeq 2$ with respect to the $\tilde{\eta}$ values shown in this figure.

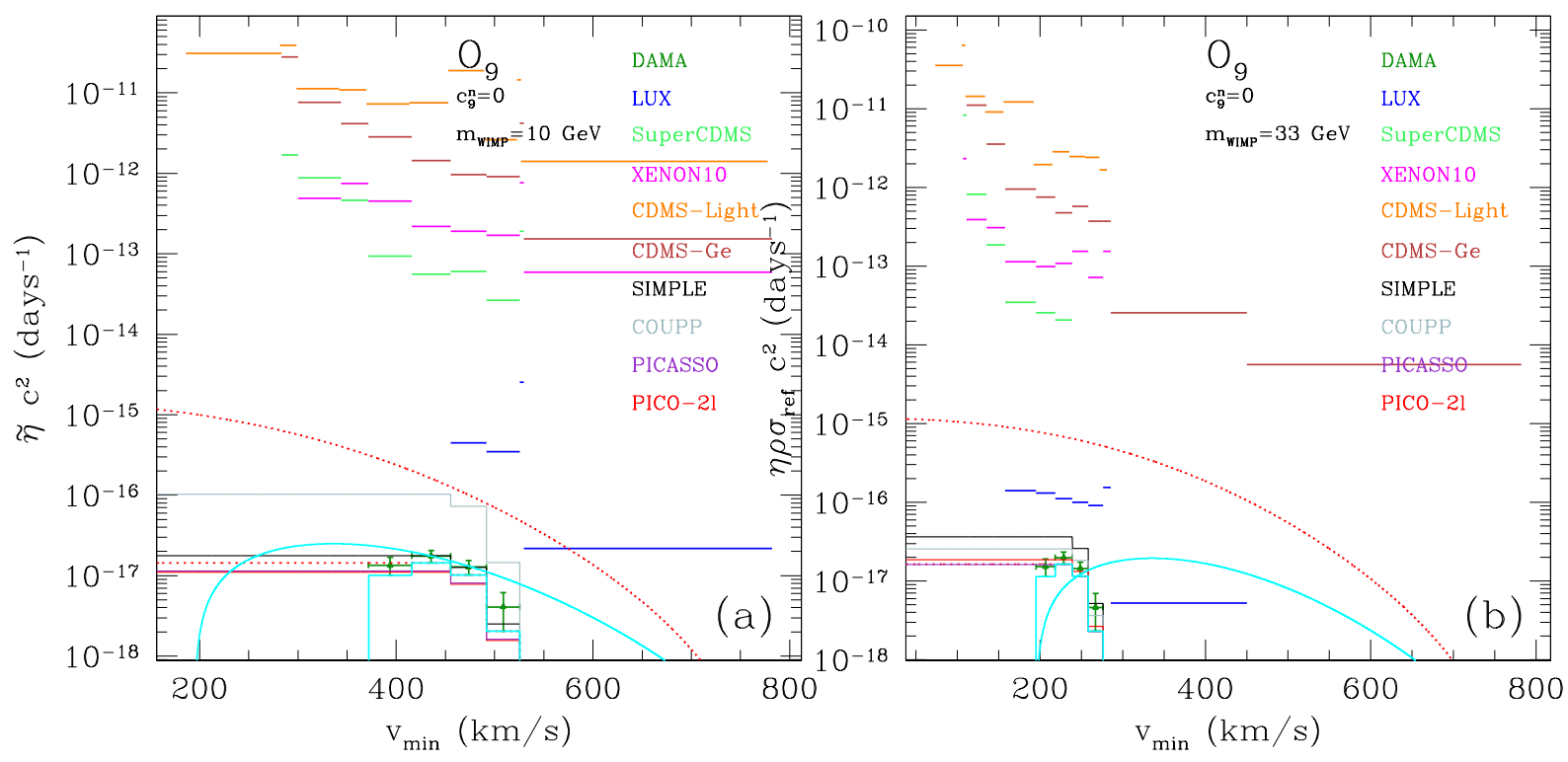

Figure 4. The same as in Figure 2 for model $\mathcal{O}_{9}$. The $\tilde{\xi}$ determinations for model $\mathcal{O}_{14}$ would be rescaled by a common factor 2 with respect to the $\tilde{\eta}$ values shown in this figure. The $\tilde{\eta}$ determinations for model $\mathcal{O}_{10}$ would be rescaled by an approximately common factor $\simeq 1 / 2$.

chambers (PICASSO, PICO-2L), which all use nuclear targets (fluorine, chlorine and iodine) with an unpaired proton.

From the phenomenological point of view we found that, although several relativistic Effective Field Theories can lead to a spin-dependent cross section, in some cases involving an explicit dependence of the scattering cross section on the WIMP incoming velocity (see 
Table 2), three main scenarios can be singled out in the non-relativistic limit which (approximately) encompass them all, and that only differ by their explicit dependence on the transferred momentum, $\left(q^{2}\right)^{n}, n=0,1,2$ : using the notation of Eq.(2.2), they are $\mathcal{O}_{4}, \mathcal{O}_{9}$ and $\mathcal{O}_{6}$, respectively.

In our quantitative analysis we pointed out that the requirement for a halo-independent analysis that one target dominates the expected rate is only needed to get estimations of the halo function from those experiments that observe an excess, but is not indispensable to get a conservative bound for those reporting a null result. We achieved this by adopting the procedure to find the minimal halo function $\tilde{\eta}^{\text {est }}\left(v_{\min }\right)$ compatible to the DAMA signal and then use it to calculate expected rates in other experiments, including droplet detectors and bubble chambers which contain several target nuclei. Using this approach we also concluded that scatterings on iodine can be assumed to be below threshold in DAMA as long as $m_{W I M P} \lesssim 60 \mathrm{GeV}$, allowing the factorization of the halo function for sodium and evading the KIMS bound (also using iodine) in the same WIMP mass range.

In particular, we found that, for $m_{W I M P} \lesssim 30 \mathrm{GeV}$ and with our assumptions on $\tilde{\eta}^{e s t}\left(v_{\text {min }}\right)$, the WIMP signal in bubble chambers and droplet detectors is dominated by scatterings off fluorine. In this case models where the expected detection rate depends on one additional factor $\left(q^{2}\right)^{n}, n>0$ show a relative enhancement of the expected rate in DAMA compared to that in fluorine detectors, with a consequent relative loss of sensitivity for the latter, because the transferred momenta $q^{2}$ which explain the DAMA modulation effect for WIMP scatterings off sodium in DAMA are larger than the corresponding ones off fluorine. In this way compatibility between DAMA and other constraints can be achieved for $\mathcal{O}_{6}$ and, to a lesser extent, for $\mathcal{O}_{9}$, but not for the standard spin-dependent scenario $\mathcal{O}_{4}$. These conclusions are only valid for a WIMP velocity distribution in the halo of our Galaxy which departs from a Maxwellian. On the other hand, for $m_{W I M P} \gtrsim 30 \mathrm{GeV}$ a strong tension between DAMA and COUPP arises for both $\mathcal{O}_{6}$ and $\mathcal{O}_{9}$ because scatterings off iodine in

COUPP become kinematically allowed, with values of the transferred momenta which are much larger than those related to fluorine and sodium.

\section{Acknowledgments}

This work was supported by the National Research Foundation of Korea(NRF) grant funded by the Korea government(MOE) (No. 2011-0024836). 


\section{A WIMP response functions}

We collect here the WIMP particle-physics response functions introduced in Eq.(2.4) and adapted from [24]:

$$
\begin{aligned}
R_{M}^{\tau \tau^{\prime}}\left(v_{T}^{\perp 2}, \frac{q^{2}}{m_{N}^{2}}\right)= & c_{1}^{\tau} c_{1}^{\tau^{\prime}}+\frac{j_{\chi}\left(j_{\chi}+1\right)}{3}\left[\frac{q^{2}}{m_{N}^{2}} v_{T}^{\perp 2} c_{5}^{\tau} c_{5}^{\tau^{\prime}}+v_{T}^{\perp 2} c_{8}^{\tau} c_{8}^{\tau^{\prime}}+\frac{q^{2}}{m_{N}^{2}} c_{11}^{\tau} c_{11}^{\tau^{\prime}}\right] \\
R_{\Phi^{\prime \prime}}^{\tau \tau^{\prime}}\left(v_{T}^{\perp 2}, \frac{q^{2}}{m_{N}^{2}}\right)= & {\left[\frac{q^{2}}{4 m_{N}^{2}} c_{3}^{\tau} c_{3}^{\tau^{\prime}}+\frac{j_{\chi}\left(j_{\chi}+1\right)}{12}\left(c_{12}^{\tau}-\frac{q^{2}}{m_{N}^{2}} c_{15}^{\tau}\right)\left(c_{12}^{\tau^{\prime}}-\frac{q^{2}}{m_{N}^{2}} c_{15}^{\tau^{\prime}}\right)\right] \frac{q^{2}}{m_{N}^{2}} } \\
R_{\Phi^{\prime \prime} M}^{\tau \tau^{\prime}}\left(v_{T}^{\perp 2}, \frac{q^{2}}{m_{N}^{2}}\right)= & {\left[c_{3}^{\tau} c_{1}^{\tau^{\prime}}+\frac{j_{\chi}\left(j_{\chi}+1\right)}{3}\left(c_{12}^{\tau}-\frac{q^{2}}{m_{N}^{2}} c_{15}^{\tau}\right) c_{11}^{\tau^{\prime}}\right] \frac{q^{2}}{m_{N}^{2}} } \\
R_{\Phi^{\prime}}^{\tau \tau^{\prime}}\left(v_{T}^{\perp 2}, \frac{q^{2}}{m_{N}^{2}}\right)= & {\left[\frac{j_{\chi}\left(j_{\chi}+1\right)}{12}\left(c_{12}^{\tau} c_{12}^{\tau^{\prime}}+\frac{q^{2}}{m_{N}^{2}} c_{13}^{\tau} c_{13}^{\tau^{\prime}}\right)\right] \frac{q^{2}}{m_{N}^{2}} } \\
R_{\Sigma^{\prime \prime}}^{\tau \tau^{\prime}}\left(v_{T}^{\perp 2}, \frac{q^{2}}{m_{N}^{2}}\right)= & \frac{q^{2}}{4 m_{N}^{2}} c_{10}^{\tau} c_{10}^{\tau^{\prime}}+\frac{j_{\chi}\left(j_{\chi}+1\right)}{12}\left[c_{4}^{\tau} c_{4}^{\tau^{\prime}}+\right. \\
\left.R_{\Sigma^{\prime}}^{\tau \tau^{\prime}}\left(v_{4}^{\tau} c_{6}^{\tau^{\prime}}+c_{6}^{\tau} c_{4}^{\tau^{\prime}}\right)+\frac{q^{4}}{m_{N}^{4}} c_{6}^{\tau} c_{6}^{\tau^{\prime}}+v_{T}^{\perp 2} c_{12}^{\tau} c_{12}^{\tau^{\prime}}+\frac{q^{2}}{m_{N}^{2}} v_{T}^{\perp 2} c_{13}^{\tau} c_{13}^{\tau^{\prime}}\right] & \frac{1}{8}\left[\frac{q^{2}}{m_{N}^{2}} v_{T}^{\perp 2} c_{3}^{\tau} c_{3}^{\tau^{\prime}}+v_{T}^{\perp 2} c_{7}^{\tau} c_{7}^{\tau^{\prime}}\right]+\frac{j_{\chi}\left(j_{\chi}+1\right)}{12}\left[c_{4}^{\tau} c_{4}^{\tau^{\prime}}+\right. \\
& \left.\frac{q^{2}}{m_{N}^{2}} c_{9}^{\tau} c_{9}^{\tau^{\prime}}+\frac{v_{T}^{\perp 2}}{2}\left(c_{12}^{\tau}-\frac{q^{2}}{m_{N}^{2}} c_{15}^{\tau}\right)\left(c_{12}^{\tau^{\prime}}-\frac{q^{2}}{m_{N}^{2}} c_{15}^{\tau \prime}\right)+\frac{q^{2}}{2 m_{N}^{2}} v_{T}^{\perp 2} c_{14}^{\tau} c_{14}^{\tau^{\prime}}\right] \\
R_{\Delta}^{\tau \tau^{\prime}}\left(v_{T}^{\perp 2}, \frac{q^{2}}{m_{N}^{2}}\right)= & \frac{j_{\chi}\left(j_{\chi}+1\right)}{3}\left(\frac{q^{2}}{m_{N}^{2}} c_{5}^{\tau} c_{5}^{\tau^{\prime}}+c_{8}^{\tau} c_{8}^{\tau^{\prime}}\right) \frac{q^{2}}{m_{N}^{2}} \\
R_{\Delta \Sigma^{\prime}}^{\tau \tau^{\prime}}\left(v_{T}^{\perp 2}, \frac{q^{2}}{m_{N}^{2}}\right)= & \frac{j_{\chi}\left(j_{\chi}+1\right)}{3}\left(c_{5}^{\tau} c_{4}^{\tau^{\prime}}-c_{8}^{\tau} c_{9}^{\tau^{\prime}}\right) \frac{q^{2}}{m_{N}^{2}} .
\end{aligned}
$$

\section{B Experimental inputs for the analysis}

In this Appendix we summarize the experimental inputs that we have used to evaluate the response function defined in Eq. (4.5) for each of the experiments included in our analysis. Whenever applicable we will follow the convention to indicate with $E_{R}$ the true recoil energy, with $E_{e e}$ the electron-equivalent energy $\left(E_{e e}=Q\left(E_{R}\right) E_{R}\right.$ with $Q$ the quenching factor $)$ and with $E^{\prime}$ the visible energy, as introduced in Section 4 . In the case of bolometric measurements we assume $Q=1$. With the exceptions of LUX we model the energy resolution with a Gaussian and we indicate the corresponding variance.

DAMA We have taken the modulation amplitudes in 0.5 keVee bins from Fig.6 of Ref.[2] (already normalized to counts/day $/ \mathrm{kg} / \mathrm{keV}$ for a total exposure of 1.17 ton $\mathrm{yr}$ ), adopting the signal region $2 \mathrm{keVee} \leq E^{\prime} \leq 4 \mathrm{keVee}$. We have adopted the value $Q_{N a}=0.3$ for the quenching factor of sodium.

LUX In the case of LUX we have assumed zero WIMP candidate events in the range $2 \mathrm{PE} \leq S_{1} \leq 30 \mathrm{PE}$ in the lower half of the signal band, as shown in Fig. 4 of Ref. [3] for the primary scintillation signal $S_{1}$ (directly in Photo Electrons, PE) for an exposure of 85.3 days and a fiducial volume of $118 \mathrm{~kg}$ of xenon. Following Ref. [30] (see Eqs. (14-15)) we have modeled the detector's response with a Poissonian fluctuation of the $S_{1}$ scintillation 
photoelectrons combined with a Gaussian resolution $\sigma_{P M T}=0.5 \mathrm{PE}$ for the photomultiplier so that the response functions defined in Eq.(4.5) are modified into:

$$
\begin{aligned}
& \mathcal{R}_{\left[E_{1}^{\prime}, E_{2}^{\prime}\right]}^{m}=\sum_{T} 8 N_{T} \mu_{T}^{2} \frac{1}{\sigma_{r e f}} \sum_{k} \sum_{\tau \tau^{\prime}} R_{m k}^{\tau \tau^{\prime}}\left[q^{2}\left(v_{\text {min }}\right)\right] \frac{W_{T k}^{\tau \tau^{\prime}}\left[y\left(v_{\text {min }}\right)\right]}{2 j_{T}+1} \times \\
& \int_{S_{1, \text { min }}}^{S_{1, \text { max }}} d S_{1} \sum_{n=1}^{\infty} \operatorname{Gauss}\left(S_{1} \mid n, \sqrt{n} \sigma_{P M T}\right) \operatorname{Poiss}\left[n, \nu\left(E_{R}\right)\right] \xi_{\text {cuts }}\left(S_{1}\right), \quad m=0,1 .
\end{aligned}
$$

In the equation above Poiss $(n, \lambda)=\lambda^{n} / n ! \exp (-\lambda)$, while $\xi_{\text {cuts }}$ represents the combination of a $50 \%$ acceptance combined with the quality cut efficiency (taken from Fig. 9 of Ref. [3]). Moreover the expected number of $\mathrm{PE}$ for a given recoil energy $E_{R}$ is given by:

$$
\nu\left(E_{R}\right)=E_{R} \times L_{e f f}\left(E_{R}\right) \times L_{y}
$$

with $L_{y}=8.8 \mathrm{PE}$. We have taken $L_{e f f}\left(E_{R}\right)$ from [31] where it is calculated including the effect of the electric field, and assumed to vanish for $S_{1}<3 \mathrm{PE}$.

SuperCDMS We include the low-energy analysis of SuperCDMS[10] with a germanium target in the energy range $1.6 \mathrm{keVnr}<E_{R}<10 \mathrm{keVnr}$ with a total exposition of $577 \mathrm{~kg}$ day and 11 observed WIMP candidates. The energy resolution is given by $\sigma_{C D M S-S i}\left(E^{\prime}\right)=$ $\sqrt{0.293^{2}+0.056^{2}\left(E^{\prime} / \mathrm{keVnr}\right)}$ in $\mathrm{keVnr}[32]$

XENON10 The analysis of XENON10 makes use of the secondary ionization signal $S_{2}$ only, with an exposition of 12.5 day and a fiducial mass of $1.2 \mathrm{~kg}$. We take the scale of the recoil energy $E_{R}$ and the recorded event spectrum in the energy range $1.4 \mathrm{keVnr}<E_{R}<$ $10 \mathrm{keVnr}$ directly from Fig. 2 of Ref. [5]. The energy resolution is given by: $\sigma_{X E N O N 10}=$ $E_{R} / \sqrt{E_{R} Q_{y}\left(E_{R}\right)}$ where $Q_{y}\left(E_{R}\right)$ is the electron yield that we calculate with the same choice of parameters as in Fig. 1 of [5].

CDMSlite CDMSlite[9] analyzes the very low range $0.170 \mathrm{keVee}<E_{e e}<7 \mathrm{keVee}$ for the electron-equivalent energy using a fiducial mass of $0.6 \mathrm{~kg}$ of germanium and an exposition of 10.3 days. We take the spectrum from Fig. 1 of Ref. [9]. We adopt the same quenching factor that we use for CoGeNT, an energy resolution $\sigma_{C D M}$ Slite $=14 \mathrm{eV}$ and the efficiency $\xi_{\text {cut }}=0.985[9]$.

CDMS-Ge We consider the data from detector T1Z5 in the range $2 \mathrm{keVnr}<E_{R}<100$ $\mathrm{keVnr}$ available in digital format from [8] with a raw exposure of $35 \mathrm{~kg}$ day on germanium target. The energy resolution is the same as in SuperCDMS, while the efficiency is taken from Fig.1 of Ref. [8].

SIMPLE The SIMPLE experiment[11] uses superheated liquid droplets homogeneously distributed in a gel to search for transitions to the gas phase produced by WIMP scatterings. The nuclear targets are made of $\mathrm{C}_{2} \mathrm{ClF}_{5}$ (for the nuclear response function of chlorine, which is not available from [24, 27], we have used a simple estimation outlined in Appendix C). SIMPLE is a threshold detector, only sensitive to the minimal deposited energy $E_{t h}$ required to trigger the nucleations, and with $E_{t h}$ controlled by the pressure of the liquid. The probability that an energy deposition $E_{R}$ on the target nucleus $T$ nucleates a droplet is given by:

$$
P_{T}\left(E_{R}\right)=1-\exp \left[-\alpha_{T} \frac{E_{R}-E_{t h}}{E_{t h}}\right]
$$




\begin{tabular}{|c|c|c|c|c|}
\hline$E_{t h}(\mathrm{keV})$ & exposure (kg day) & expected background (events) & measured events & $95 \%$ upper bound \\
\hline 7.8 & 55.8 & 0.8 & 2 & 5.92 \\
11 & 70 & 0.7 & 3 & 8.26 \\
15.5 & 311.7 & 3 & 8 & 12.29 \\
\hline
\end{tabular}

Table 3. For each operating threshold used in COUPP we provide the corresponding exposure, expected background, number of measured events and 95\% C.L. upper bound obtained with the Feldman-Cousin method [33] used in our analysis

\begin{tabular}{|c|c|}
\hline$E_{t h}(\mathrm{keV})$ & $95 \%$ upper bound \\
\hline 1.7 & 1.1 \\
2.9 & 1.5 \\
4.1 & 11 \\
5.8 & 9 \\
6.9 & 1.3 \\
16.3 & 3.1 \\
39 & 1.5 \\
55 & 6 \\
\hline
\end{tabular}

Table 4. 95\% C.L. upper bounds (extracted from Fig. 5 of Ref.[13]) for each operating threshold used in PICASSO.

where $\alpha_{T}$ is determined by fitting calibrations with neutron sources. With an exposure of $6.71 \mathrm{~kg}$ day and $E_{t h}=7.8$ SIMPLE observed 1 event, consistent to an expected background of 2.2. This can be converted to an upper bound of 3.16 events using the Feldman-Cousin method [33]. We use $\alpha_{F}=\alpha_{C}=3.6$.

COUPP The COUPP experiment[12] searches for WIMPs using nucleations in a bubble chamber and is also a threshold detector. In the case of COUPP the target material is $C F_{3} I$. For each operating threshold used in COUPP the corresponding exposure, expected background, number of measured events and 95\% C.L. upper bound obtained with the Feldman-Cousin method [33] used in our analysis are summarized in Table 3. We adopt the nucleation probability (B.3) with $\alpha_{F}=\alpha_{C}=0.15$, while for iodine we assume $P_{I}=1$, corresponding to $\alpha_{I} \rightarrow \infty$ in (B.3).

PICASSO The Picasso experiment[13] is a bubble chamber using $C_{3} F_{8}$, operated with eight energy thresholds. For each of the latter we provide the corresponding upper bound on the number of events (normalized to events $/ \mathrm{kg} /$ day) in Table 4 (extracted from Fig. 5 of Ref.[13]). We use the nucleation probability of Eq.(B.3) with $\alpha_{C}=\alpha_{F}=5$.

PICO-2L The PICO-2L collaboration operated a $C_{3} F_{8}$ bubble chamber experiment with four energy thresholds. For each of them we provide the corresponding exposure, number of measured events and 95\% C.L. upper bound (conservatively assuming zero background) used in our analysis in Table 5. In particular we conservatively chose to use the raw data without the subtraction adopted in [14] which makes use of time correlations among measured events. We adopt the nucleation probability (B.3) with $\alpha_{F}=\alpha_{C}=0.15$. 


\begin{tabular}{|c|c|c|c|}
\hline$E_{\text {th }}(\mathrm{keV})$ & exposure (kg day) & measured events & 95\% upper bound \\
\hline 3.2 & 74.8 & 9 & 16.77 \\
4.4 & 16.8 & 0 & 3.09 \\
6.1 & 82.2 & 3 & 8.25 \\
8.1 & 37.8 & 0 & 3.09 \\
\hline
\end{tabular}

Table 5. For each operating threshold used in PICO-2L we provide the corresponding exposure, number of measured events and 95\% C.L. upper bound (assuming zero background) used in our analysis.

\section{Nuclear response functions for chlorine}

In the case of chlorine a shell model calculation for the nuclear response functions $W_{\Sigma^{\prime \prime}}^{\tau \tau^{\prime}}$ and $W_{\Sigma^{\prime}}^{\tau \tau^{\prime}}$ is not available, so we assume $W_{\Sigma^{\prime}}^{\tau \tau^{\prime}}=2 W_{\Sigma^{\prime \prime}}^{\tau \tau^{\prime}}$ and use a Gaussian approximation for the $q^{2}$ dependence. In particular, combining the usual spin-dependent scaling law written as $[28]$ :

$$
S(0)=\frac{1}{\pi} \frac{\left(2 j_{T}+1\right)\left(j_{T}+1\right)}{j_{T}}\left(a_{p}<S_{p}>+a_{n}<S_{n}>\right)^{2}
$$

with the Gaussian form factor [34]:

$$
\frac{S\left(q^{2}\right)}{S(0)}=e^{-q^{2} R^{2} / 4}, \quad R=\left(0.92 A_{T}^{1 / 3}+2.68-0.78 \sqrt{\left(A_{T}^{1 / 3}-3.8\right)^{2}+0.2}\right) \mathrm{fm},
$$

(where $A_{T}$ is the target nucleus mass number) the identities of Eq.(2.7) imply:

$$
\begin{aligned}
& W_{\Sigma^{\prime \prime}}^{\tau \tau^{\prime}}\left(q^{2}\right)=\frac{8}{3 \pi} \frac{\left(2 j_{T}+1\right)\left(j_{T}+1\right)}{j_{T}}<S^{\tau}><S^{\tau^{\prime}}>e^{-q^{2} R^{2} / 4} \\
& W_{\Sigma^{\prime}}^{\tau \tau^{\prime}}\left(q^{2}\right)=\frac{4}{3 \pi} \frac{\left(2 j_{T}+1\right)\left(j_{T}+1\right)}{j_{T}}<S^{\tau}><S^{\tau^{\prime}}>e^{-q^{2} R^{2} / 4},
\end{aligned}
$$

with $<S^{0}>=\left(<S_{p}>+<S_{n}>\right) / 2$ and $<S^{1}>=\left(<S_{p}>-<S_{n}>\right) / 2$. For both ${ }^{35} \mathrm{Cl}$ and ${ }^{37} \mathrm{Cl}$ we adopt $\left\langle S_{p}>=-0.051\right.$ and $\left\langle S_{p}\right\rangle=-0.0088$ [35].

\section{References}

[1] P. A. R. Ade et al. [Planck Collaboration], Astron. Astrophys. 571, A16 (2014) [arXiv:1303.5076 [astro-ph.CO]].

[2] R. Bernabei et al. [DAMA and LIBRA Collaborations], Eur. Phys. J. C 67, 39 (2010) [arXiv:1002.1028 [astro-ph.GA]].

[3] D. S. Akerib et al. [LUX Collaboration], Phys. Rev. Lett. 112, no. 9, 091303 (2014) [arXiv:1310.8214 [astro-ph.CO]].

[4] E. Aprile et al. [XENON100 Collaboration], Phys. Rev. Lett. 109, 181301 (2012) [arXiv:1207.5988 [astro-ph.CO]].

[5] J. Angle et al. [XENON10 Collaboration], Phys. Rev. Lett. 107, 051301 (2011) [Erratum-ibid. 110, 249901 (2013)] [arXiv:1104.3088 [astro-ph.CO]]. 
[6] S. C. Kim, H. Bhang, J. H. Choi, W. G. Kang, B. H. Kim, H. J. Kim, K. W. Kim and S. K. Kim et al., Phys. Rev. Lett. 108, 181301 (2012) [arXiv:1204.2646 [astro-ph.CO]].

[7] Y. Kim, talk given at $13^{t} h$ International Conference on Topics in Astroparticle and Underground Physics, September 8-13 2013, Asilomar, California USA (TAUP2013).

[8] Z. Ahmed et al. [CDMS-II Collaboration], Phys. Rev. Lett. 106, 131302 (2011) [arXiv:1011.2482 [astro-ph.CO]].

[9] R. Agnese et al. [SuperCDMS Soudan Collaboration], Phys. Rev. Lett. 112, 041302 (2014) [arXiv:1309.3259 [physics.ins-det]].

[10] R. Agnese et al. [SuperCDMS Collaboration], arXiv:1402.7137 [hep-ex].

[11] M. Felizardo, T. A. Girard, T. Morlat, A. C. Fernandes, A. R. Ramos, J. G. Marques, A. Kling and J. Puibasset et al., Phys. Rev. Lett. 108, 201302 (2012) [arXiv:1106.3014 [astro-ph.CO]].

[12] E. Behnke et al. [COUPP Collaboration], Phys. Rev. D 86, no. 5, 052001 (2012) [Erratum-ibid. D 90, no. 7, 079902 (2014)] [arXiv:1204.3094 [astro-ph.CO]].

[13] S. Archambault et al. [PICASSO Collaboration], Phys. Lett. B 711, 153 (2012) [arXiv:1202.1240 [hep-ex]].

[14] C. Amole et al. [SNO Collaboration], arXiv:1503.00008 [astro-ph.CO].

[15] J. H. Lee, G. B. Kim, I. S. Seong, B. H. Kim, J. H. Kim, J. Li, J. W. Park and J. K. Lee et al., Nucl. Instrum. Meth. A 782, 133 (2015) [arXiv:1502.03800 [physics.ins-det]].

[16] P. J. Fox, J. Liu and N. Weiner, Phys. Rev. D 83, 103514 (2011) [arXiv:1011.1915 [hep-ph]].

[17] C. McCabe, Phys. Rev. D 84, 043525 (2011) [arXiv:1107.0741 [hep-ph]]; M. T. Frandsen, F. Kahlhoefer, C. McCabe, S. Sarkar and K. Schmidt-Hoberg, JCAP 1201, 024 (2012) [arXiv:1111.0292 [hep-ph]].

[18] P. Gondolo and G. B. Gelmini, JCAP 1212, 015 (2012) [arXiv:1202.6359 [hep-ph]]; E. Del Nobile, G. B. Gelmini, P. Gondolo and J. H. Huh, JCAP 1310, 026 (2013) [arXiv:1304.6183 [hep-ph]]; JCAP 1403, 014 (2014) [arXiv:1311.4247 [hep-ph]].

[19] S. Scopel and K. Yoon, JCAP 1408, 060 (2014) [arXiv:1405.0364 [astro-ph.CO]].

[20] P. Ullio, M. Kamionkowski and P. Vogel, JHEP 0107, 044 (2001) [hep-ph/0010036].

[21] E. Del Nobile, G. B. Gelmini, A. Georgescu and J. H. Huh, arXiv:1502.07682 [hep-ph].

[22] M. Freytsis and Z. Ligeti, Phys. Rev. D 83, 115009 (2011) [arXiv:1012.5317 [hep-ph]].

[23] C. Arina, E. Del Nobile and P. Panci, Phys. Rev. Lett. 114, 011301 (2015) [arXiv:1406.5542 [hep-ph]].

[24] A. L. Fitzpatrick, W. Haxton, E. Katz, N. Lubbers and Y. Xu, JCAP 1302, 004 (2013) [arXiv:1203.3542 [hep-ph]]; N. Anand, A. L. Fitzpatrick and W. C. Haxton, Phys. Rev. C 89, no. 6, 065501 (2014) [arXiv:1308.6288 [hep-ph]].

[25] V. Cirigliano, M. L. Graesser and G. Ovanesyan, JHEP 1210, 025 (2012) [arXiv:1205.2695 [hep-ph]]; V. Cirigliano, M. L. Graesser, G. Ovanesyan and I. M. Shoemaker, Phys. Lett. B 739, 293 (2014) [arXiv:1311.5886 [hep-ph]].

[26] S. Scopel and J. H. Yoon, Phys. Rev. D 91, no. 1, 015019 (2015) [arXiv:1411.3683 [hep-ph]].

[27] R. Catena and B. Schwabe, arXiv:1501.03729 [hep-ph].

[28] J. Engel, S. Pittel and P. Vogel, Int. J. Mod. Phys. E 1, 1 (1992).

[29] N. Fornengo and S. Scopel, Phys. Lett. B 576, 189 (2003) [hep-ph/0301132].

[30] E. Aprile et al. [XENON100 Collaboration], Phys. Rev. D 84, 052003 (2011) [arXiv:1103.0303 [hep-ex]]. 
[31] http://luxdarkmatter.org/talks/20131030_LUX_First_Results.pdf

[32] Z. Ahmed et al. [CDMS Collaboration], Phys. Rev. D 81, 042002 (2010) [arXiv:0907.1438 [astro-ph.GA]].

[33] G. J. Feldman and R. D. Cousins, Phys. Rev. D 57, 3873 (1998) [physics/9711021 [physics.data-an]].

[34] G. Belanger, F. Boudjema, A. Pukhov and A. Semenov, Comput. Phys. Commun. 180, 747 (2009) [arXiv:0803.2360 [hep-ph]].

[35] M. T. Ressell, M. B. Aufderheide, S. D. Bloom, K. Griest, G. J. Mathews and D. A. Resler, Phys. Rev. D 48, 5519 (1993). 